\title{
Análisis y evaluación de las alternativas de protección de las aguas subterráneas durante las operaciones de extracción de los residuos (alquitranes ácidos) en una laguna de Arganda del Rey (Madrid)
}

\author{
D. Barettino Fraile (1), T. García Ruiz ${ }^{(2)}$, R. Juncosa Rivera ${ }^{(3)}$, P. Mora Peris ${ }^{(4)}$, E. Prendes \\ Alvargonzález ${ }^{(5)}$ y. J. M. Salve Sánchez ${ }^{(6)}$
}

(1) Dirección General Economía Circular. Consejería de Medio Ambiente, Vivienda y Agricultura. Comunidad de Madrid, C/ Alcalá, 16. $3^{\mathrm{a}}$ planta, 28014 Madrid. daniel.barettino@ madrid.org

(2) Compañía General de Soporte a la Ingeniería S.L. (CGSi), C/Blasco de Garay n 76, 28015 Madrid. t.garcia@cgsingenieria.com

(3) ETSI Caminos, Canales y Puertos, Universidad de La Coruña. Campus de Elviña s/n, 15091 La Coruña. ricardo.juncosa@udc.es

(4) Universidad Politécnica de Madrid, Calle de Ríos Rosas, 21, 28003 Madrid. pedro.mora@upm.es (5) Experto contratado por el GrupoTRAGSA. Ctra. A-42, km 6.8, 28916 Leganés (Madrid). eprendes.ext@tragsa.es (6) Consultor, C/ Greco, 2, 28450 Collado Mediano (Madrid). juanmanuelsalve@gmail.com

\begin{abstract}
RESUMEN
La Comunidad de Madrid está llevando a cabo la restauración integral de una antigua gravera contaminada con residuos peligrosos, mayoritariamente sulfonatos de petróleo (alquitranes ácidos), procedentes del tratamiento de aceites industriales usados.

Parte de dichos vertidos, realizados en las décadas de los años 1970 y 1980, se depositaron en una laguna originada por la explotación de áridos por debajo del nivel piezométrico del acuífero, conformando una balsa con $50000 \mathrm{~m}^{3}$ de residuos peligrosos. La diversa ubicación, naturaleza y estado de los residuos requiere diferentes soluciones para su extracción y tratamiento, por lo que el proyecto de restauración consta de varias fases. Los residuos depositados producen un efecto de confinamiento en el acuífero, dado que la cota del fondo de los residuos es inferior al nivel piezométrico. Con estos condicionantes es crítico establecer un procedimiento de protección de las aguas subterráneas a la hora de acometer la próxima fase, consistente en la extracción de los residuos situados por debajo del nivel piezométrico.

En este artículo se describe el proceso que se ha llevado a cabo para seleccionar la mejor alternativa desde el punto de vista hidrogeológico, considerando todos los datos disponibles obtenidos en las fases previas del proyecto. Una vez planteadas y caracterizadas todas las alternativas posibles se ha seleccionado de forma argumentada y razonada como mejor alternativa la realización de una barrera impermeabilizante perimetral a la balsa y empotrada en una capa de arcillas de $2 \mathrm{~m}$ de espesor situada en el subsuelo de la balsa.
\end{abstract}

Palabras clave: acuífero, aguas subterráneas, gravera, recuperación de residuos, restauración.

\section{Analysis and evaluation of groundwater protection alternatives during waste extraction operations (acid tars) in a lagoon in Arganda del Rey (Madrid)}

ABSTRACT

The Community of Madrid is carrying out the integral restoration of an old gravel pit contaminated with hazardous waste, mostly petroleum sulphonates (acid tars), from the treatment of used industrial oil. Part of these discharges, from the 1970s and 1980s, were deposited in a lagoon generated by the exploitation of aggregates below the piezometric level of the aquifer, forming a raft with 50,000 $\mathrm{m} 3$ of hazardous waste. The different locations, nature and conditions of the waste require different solutions for its extraction and 
treatment, so the restoration project consists of several phases.

The deposited waste produces an effect of confinement in the aquifer, since the bottom level of the waste is lower than the piezometric level. With these conditions, it is critical to establish a procedure for the protection of the groundwater when executing the next phase, consisting of the extraction of waste located below the piezometric level.

This article describes the process that has been carried out to select the best alternative from a hydrogeological point of view, considering all the available data obtained in the previous phases of the project. Once all the possible alternatives had been considered and characterized in a reasoned way, we chose the construction of a perimeter barrier around the raft embedded in a clay layer of $2 \mathrm{~m}$ thick located in the base of the raft as the best option.

Keywords: aquifer; groundwater; gravel; restoration; waste recovery.

\section{ABRIDGED ENGLISH VERSION}

\section{Introduction and methodology}

The Community of Madrid acquired a plot of 64,000 $\mathrm{m}^{2}$ in 2008 to decontaminate an old gravel pit where hazardous waste from the petroleum sulphonate (acid tar) that had been spilled there (Fig. 1). These spills had accumulated in an old lagoon, previously caused by the exploitation of aggregates. As shown in Figure 1 , in the plot acquired by the Community of Madrid, three zones that require different solutions can be distinguished according to their current state:

- Main lagoon with fluid residues where the viscosity increases with depth.

- Southeast lagoon with semi-solid waste.

- Soils contaminated with residues of different types.

A volume of about $50,000 \mathrm{~m}^{3}$ of oily sulphonates (acid tar) in the main lagoon is estimated and 10,000 $\mathrm{m}^{3}$ in the southeast lagoon. The estimated total volume of contaminated soil is $33,000 \mathrm{~m}^{3}$.

As the working site is an old gravel pit a large amount of the volume of waste is located below the water table, which is an important negative factor that must be taken into account to avoid any impact on the aquifer or the groundwater. Because of this serious situation the Community of Madrid took the decision to develop an ambitious restoration action plan of the area. The main objective of the study presented in this article is to select the most suitable option for the protection of groundwater during the mechanical extraction phase of the residues detected at the bottom of the main raft. This extraction work is framed within the extraction Phase 1.B of the plan.

Figure 2 shows the evolution of the previous extraction activities of Phase 1.A corresponding to the extraction of the transportable material by pumping. This graph shows an adverse factor, from the impact point of view, to the underlying aquifer during the extraction of the bottom residue activities, since the bottom level of the residues (522.5 $\mathrm{m}$ a.s. I.) is located below the piezometric level (525-526 $m$ a.s. I.). Taking into account all the above, it is essential to have planned and implemented what is considered to be the best option for the hydrogeological control of the opera- tions of Phase $1 . B$, before the beginning it and with an exhaustive follow-up during the entire development process. The operations of Phase 1.B pose a great challenge, not only in terms of the extraction of the waste below the piezometric level of the aquifer but also the selection and design of corrective activities and hydrogeological controls, as there are few similar studies available, not only in Spain, but worldwide. It is necessary that the solution adopted be justified with criteria argued in a reasoned and quantitative way.

As shown in Figure 3, the lagoon is located within the Southeast Regional Park, which is an added problem, due to its proximity to wetlands of great ecological interest. Figure 4 shows comparatively the corresponding study area, at the beginning of the discharge in 1975 and at the start of the actions by the Community of Madrid in 2014. Figure 5 indicates the layers of waste identified in the lagoon and in Table 1 their physical and chemical characteristics are summarized, according to the investigations carried out. Figure 6 shows the presence of a layer of silt under the waste and a geological section of the raft area according to previous studies.

The hydrogeological control presented in this article was carried out in 2017, prior to the beginning of Phase $1 . B$, in order to plan this phase well in advance. Figures 7 and 8 show the surface mass location of interest in the raft surroundings and Table 2 summarizes the result of in situ measurements in three lagoons close to the raft of the study. From the hydrogeological point of view the raft is located in the groundwater body 030.007: Aluviales: Jarama-Tajuña (Fig. 9). A complete hydrogeological characterization of the area has been carried out, consulting different water point databases of representative organisms, as shown in Figure 10.

Figure 11 shows the research and control drillings on the plot. During the months of May, June and July 2017 piezometric measurements were taken, which allowed the establishment of the underground flow network, as well as the main directions of the water circulation flow underground (Fig. 12). Groundwater samples were also taken at different points for laboratory analysis. 
Table 3 shows the analytical results of the water in the surrounding lagoons and their comparison with the applicable legislation.

To find out the values of the hydrodynamic parameters, several pumping tests were carried out at the points inside the plot indicated in Figures 13 and 14 show an example of the evolution and interpretation of a pumping test. Tables 4 and 5 show the results obtained in the tests. Figure 15 shows the adjustment of the piezometric decreases observed for the graphic estimation of the radius of influence.

Modelling has been carried out using the MODFLOW code where the objective is to obtain quantitative data that allow an analysis of groundwater management alternatives during the operations of Phase 1.B, with the conceptual model and boundary conditions indicated in Figures 16 and 17 and according to the different hypotheses shown in Figure 18.

\section{Results and discussion}

Figure 19 shows the alternatives that have been identified for the hydrogeological control of the aquifer during the operations of waste extraction from the raft by mechanical means in Phase 1.B.

Figure 20 shows the underground flow conditions in the case of drainage or direct bilge with a pump from the raft bottom. This system is totally inadvisable due to the high piezometric gradients that can originate siphoning effects with dragging, breaking and changes in the bearing capacity of the land. The extraction of waste below a water table is also discouraged due to the lack of direct control of the extractive operations generated by many unknown factors, some of which are difficult to forecast and quantify.

The reduction of piezometric levels through wells or ditches has been studied with MODFLOW, under different hypotheses. The linear drainage system is presented in Figures 21, 22 and 23 and the perimeter drainage system in Figure 24. Table 6 summarizes the flow and volume values obtained in the simulation with MODFLOW.

Table 6 shows the high magnitude of the flows and volumes required for drainage through pumping wells or with trenches, regardless of the device used. This fact is a problem when working in dry conditions due to the volume of groundwater to be extracted, the effect caused by the dragging of undesirable substances during drainage and the quality standards required by current legislation for the reincorporation of extracted groundwater into the water environment.

Regarding the option of a waterproof screen construction, which is presented in Figure 25, and in Table 7 the calculated results of groundwater extraction are summarized, under the hypothesis of the waterproofness of the system with the screen embedded in the underlying clay layer, with a daily evaporation of $5 \mathrm{~mm}$ per day, the theoretical volume that could be eliminated by evaporation for an area of $2,000 \mathrm{~m} 2$ would therefore be $10 \mathrm{~m} 3$ per day.

Once the identification and characterization of the options have been carried out, a matrix has been prepared for decision making (Table 8), in which the advantages and disadvantages of each option is shown for a reasoned decision based on logical criteria. The most advantageous option considered is the construction of a sealed perimeter barrier. This waterproofing system by means of a sealed screen is the most suitable for the control of groundwater during the operations to extract the waste on the bottom raft in Phase 1.B. As the elimination by evaporation in the outer raft of the pumped water is technically possible, this system, although it is expensive, represents a very important saving in the concepts of pumping, treatment and discharge of underground water to public channels. Once the waterproof barrier anchored in the clay is finished, it is necessary to build the evacuation and drainage wells and the corresponding piezometers within it to verify the waterproofness of the system. During the extraction operations of Phase 1.B. piezometric and quality control is necessary in the perimeter network and adjacent lagoons, as well as in the wells and piezometers of the control barrier.

\section{Introducción}

Con el fin de restaurar una parcela de 6.41 hectáreas en el Término Municipal de Arganda del Rey (Madrid) la Comunidad de Madrid adquirió la propiedad en el año 2008. Esta parcela se encuentra dentro del Parque Regional del Sureste. En la décadas del 70 y 80 se vertieron en esta sulfonatos de petróleo (mayoritariamente alquitranes ácidos) procedentes de la firma Ulibarri, S.A., sociedad ya extinguida cuya actividad consistía en la regeneración de aceites industriales usados.

Parte de dichos vertidos, se depositaron en una laguna originada por la explotación de áridos por debajo del nivel freático, conformando una balsa de residuos peligrosos de $12750 \mathrm{~m}^{2}$ de superficie y una profundidad máxima de $7.5 \mathrm{~m}$.

Según se muestra en la Figura 1, en la parcela se pueden distinguir, en función de su estado actual, tres zonas que requieren diferentes soluciones:

- Laguna principal con residuos en estado fluido cuya viscosidad aumenta con la profundidad, con un volumen de unos $50000 \mathrm{~m}^{3}$ de sulfonatos de petróleo oleosos.

- Laguna sureste con unos $10000 \mathrm{~m}^{3}$ de residuos en estado semisólido.

- Suelos contaminados con residuos de diversa tipología, en un volumen de unos $33000 \mathrm{~m}^{3}$.

Es muy importante tener en cuenta que, dado que el emplazamiento se trata de una antigua gravera, gran parte del volumen de residuos se encuentra situado bajo el nivel freático, lo que supone un factor negativo muy importante que es preciso controlar de cara a evitar la contaminación del acuífero. 
Para evitar de modo permanente la transmisión de contaminantes a las aguas subterráneas, se decidió eliminar el pasivo ambiental, planteando para ello diferentes soluciones y métodos para tratar los materiales contaminantes, los suelos contaminados y restaurar el medio ambiente con el fin de obtener una descontaminación completa del lugar y lograr una recuperación de la biodiversidad. del nivel piezométrico y valoración energética de los mismos.

- Fase 1B se realizará la extracción de los residuos más viscosos que se encuentran en la parte más profunda de la laguna principal y de la laguna sureste.

Hasta el momento se han extraído mediante bombeo y valorizado energéticamente 42346.86 toneladas

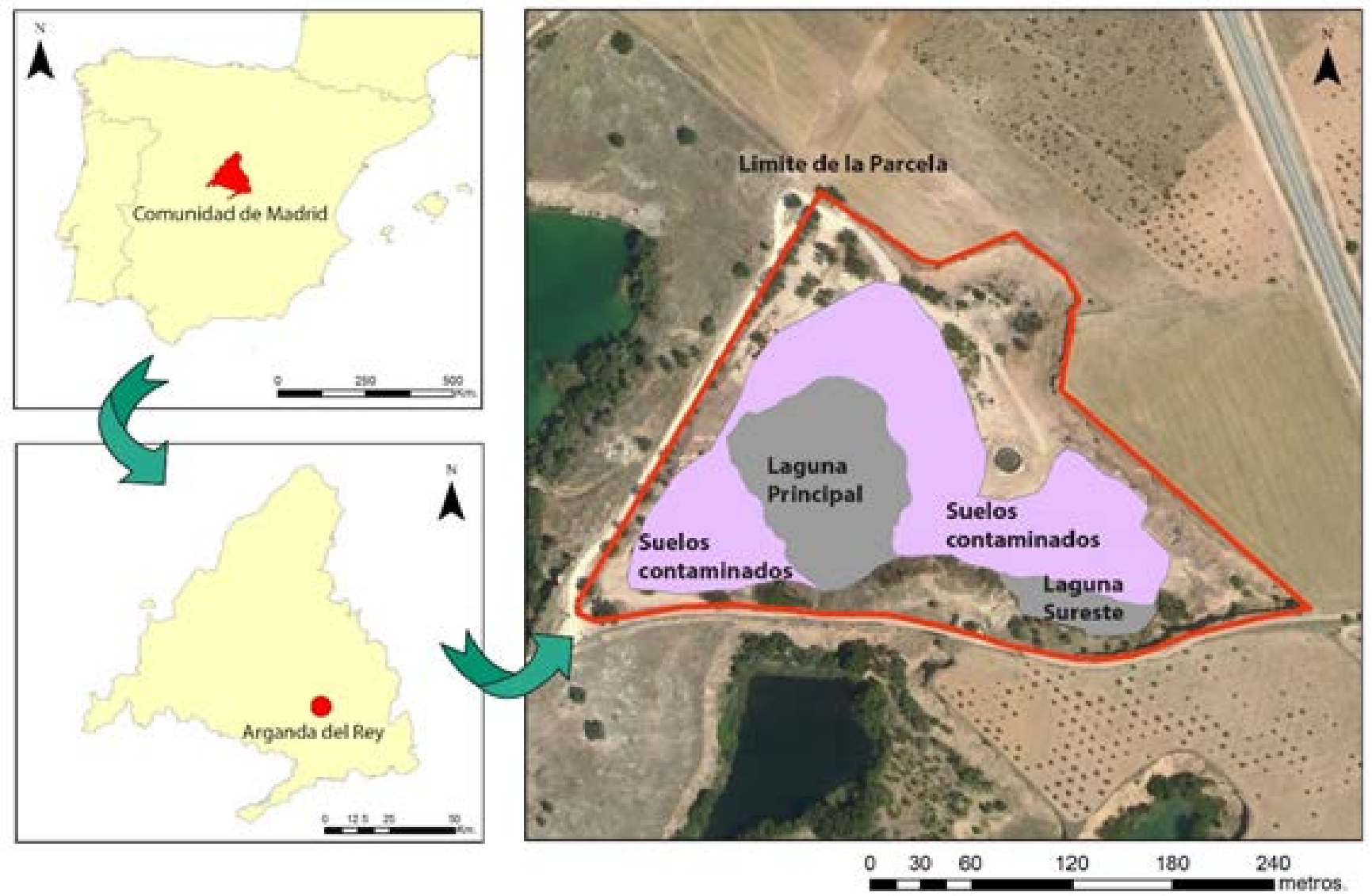

Figura 1. Ubicación del área de estudio y distribución en la parcela de la Laguna principal, la Laguna SE y los suelos contaminados (elaboración propia en base a la cartografía del IGN y a las imágenes del PNOA).

Figure 1. Location of the study area and distribution in the plot of the main lagoon, the SE lagoon and the contaminated soils (own elaboration based on the mapping of IGN and PNOA images).

La directriz de todas estas actuaciones es la eliminación total del pasivo ambiental, mediante la retirada completa del foco contaminante, tanto en la balsa como en los suelos contaminados, con el objetivo final de uso público del espacio en el contexto del Parque Regional.

El proyecto de recuperación ambiental se ha planteado como una actuación estructurada en tres fases. La primera de ellas consiste en la extracción, transporte, tratamiento y valorización energética de los residuos de las lagunas principal y sureste. La segunda tiene como fin la descontaminación de los suelos y la tercera la restauración ecológica de los terrenos.

La primera fase se ha dividido en dos:

Fase $1 \mathrm{~A}$ se realizó la extracción por bombeo de la fracción de residuos más fluidos por encima de residuos de la laguna principal en la Fase 1A. A partir de este momento, hay que abordar la siguiente etapa, la Fase 1B, consistente en la extracción de los residuos que se encuentran por debajo del nivel piezométrico del acuífero.

Una descripción extensa de la caracterización del emplazamiento y del análisis y selección de alternativas para su recuperación ambiental se encuentra en Mora Peris et al., 2017.

En la Figura 2 se presenta la evolución de las actividades extractivas del material extraíble por bombeo que, desde su comienzo en 2015 , han avanzado a un ritmo importante reduciendo el espesor de los materiales contaminantes ubicados en la balsa en más de $3 \mathrm{~m}$ de espesor a finales de 2018. 
Cota m Cotas: Nivel Balsa-Nivel Freático

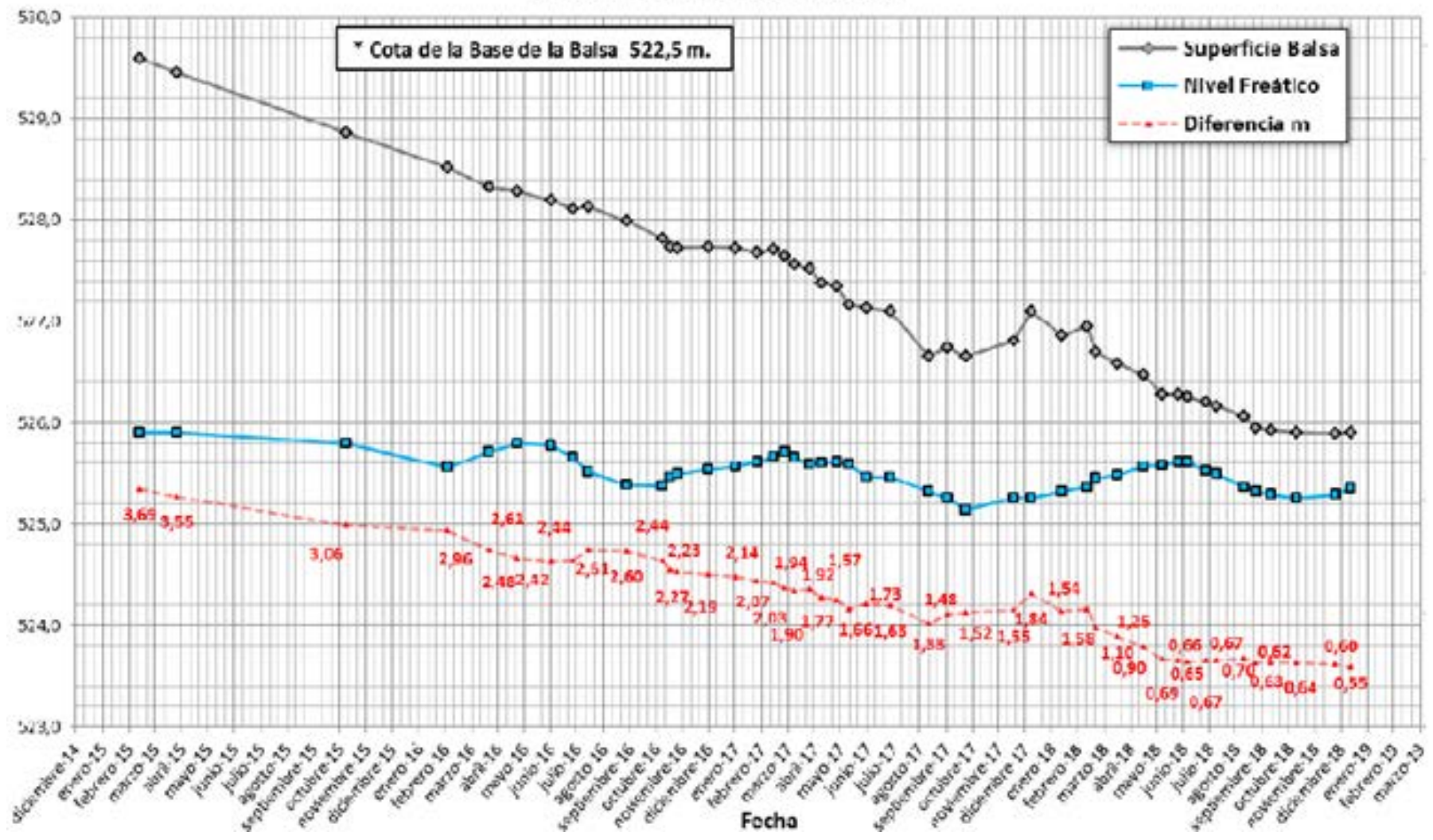

Figura 2. Evolución de las operaciones de la Fase $1 \mathrm{~A}$ del material extraíble por bombeo.

Figure 2. Evolution of the operations of Phase 1A of the removable material by pumping.

La cota topográfica de la superficie de capa del material contaminante depositada en la balsa ha evolucionado en este periodo desde la cota aproximada de 529.6 a 525.9 m s.n.m.

En dicha figura puede observarse también que la cota del nivel piezométrico en la zona de la balsa ha fluctuado en el periodo $2015-2018$ entre los 525 y 526 m s.n.m. Además, a finales del año 2018 hay tan sólo una diferencia de unos $0.6 \mathrm{~m}$ entre la cota de la lámina del residuo y el nivel piezométrico.

La cota de fondo de los residuos (522.5 m s.n.m.) se encuentra por debajo de la cota piezométrica (525-526 m s.n.m.). Lo que significa que en el área de la balsa el acuífero está en carga, confinado por los propios residuos, por lo que conforme se vaya realizando la extracción de los residuos por medios mecánicos podrán producirse aportes del agua subterránea a la balsa si no se toman las medidas correctoras adecuadas. Estos aportes podrían comenzar de forma incipiente tan pronto se alcance la cota piezométrica, e irían incrementándose a lo largo de la excavación hasta el fondo de la balsa.

Por todo lo anterior es imprescindible tener prevista e implementada la que se considere la mejor alternativa para el control hidrogeológico de las operaciones de la Fase 1B, antes del comienzo de la misma y realizar un seguimiento exhaustivo durante todo el proceso de su desarrollo.

\section{Objetivos}

El objetivo fundamental es presentar los trabajos realizados para seleccionar la alternativa más adecuada para la protección de las aguas subterráneas durante la fase de extracción por medios mecánicos de los residuos ubicados en el fondo de la balsa principal, que, dada su densidad y viscosidad, no se pueden bombear y que se encuentran situados por debajo del nivel piezométrico.

Hay que destacar que las operaciones de la Fase 1B suponen un gran desafío, tanto en lo que se refiere a la extracción de residuos por debajo de la cota piezométrica, como a la selección y diseño de las actividades correctivas y control hidrogeológico, debido a las escasas experiencias de casos de estudio similares con las que se cuenta, no sólo en España, sino a nivel mundial. Por ello, es preciso que la solución adoptada sea justificada con criterios científicos y técnicos bien argumentados de forma cualitativa y cuantitativa con una razonada valoración multidisciplinar del problema.

\section{Zona de estudio}

Previamente al comienzo en 2015 de la Fase 1A de extracción de los residuos se efectúo un completo y detallado estudio de la parcela con la realización de sondeos, calicatas, muestreos, geofísica, análisis de agua, 
etc. El resultado de estos trabajos permitió una caracterización exhaustiva, tanto del material depositado en la balsa, como de la geología del subsuelo de la parcela (TRAGSA and EMGRISA, 2013; Mora Peris et al., 2017).

La laguna donde se hallan los residuos a extraer se encuentra dentro del Parque Regional del Sureste (Figura 3), lo que supone una problemática añadida, dada su proximidad a zonas de humedales de gran interés ecológico.

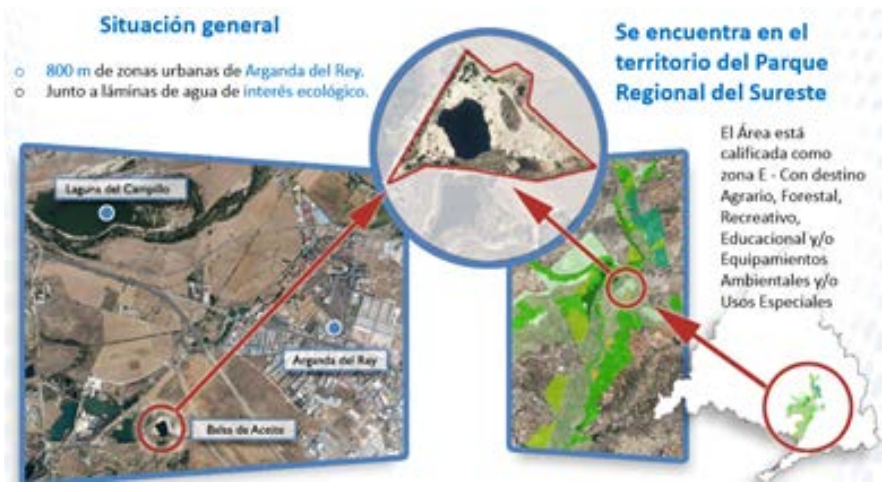

Figura 3. Situación de la Laguna de Arganda del Rey dentro del Parque Regional del Sureste.

Figure 3. Situation of the Laguna de Arganda del Rey within the Southeast Regional Park.

En la Figura 4 se puede observar de forma comparativa los estados de la zona de estudio correspondientes, respectivamente, al comienzo del vertido en 1975 y al inicio de las actuaciones por parte de la Comunidad de Madrid en 2014. Nótese que la disminución de la superficie se debe al vertido de residuos sólidos sobre los alquitranes ácidos.
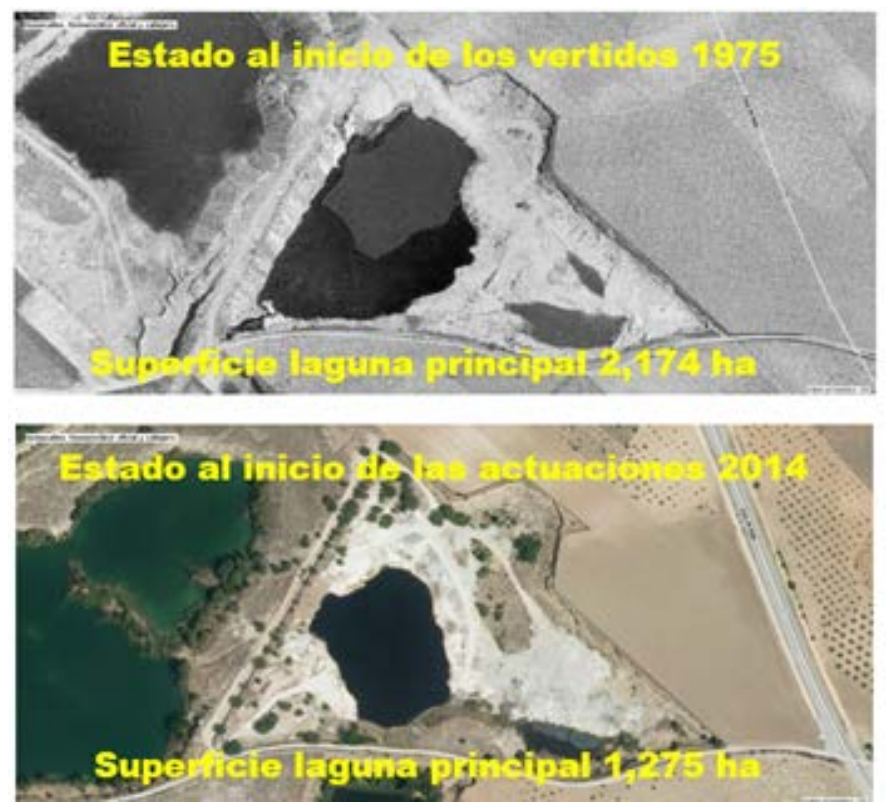

Figura 4. Estado de la laguna al inicio del vertido en 1975 y al inicio de las actuaciones por parte de la Comunidad de Madrid en 2014. Figure 4. Status of the lagoon at the beginning of the discharge in 1975 and at the beginning of the Community of Madrid actions in 2014.
Los estudios técnicos de investigación y caracterización de la parcela realizados entre los años 2009 y 2013, revelaron la existencia en la laguna principal de cuatro niveles o estratos de residuos diferenciados en la balsa, capas A, B, C y D (Figura 5).
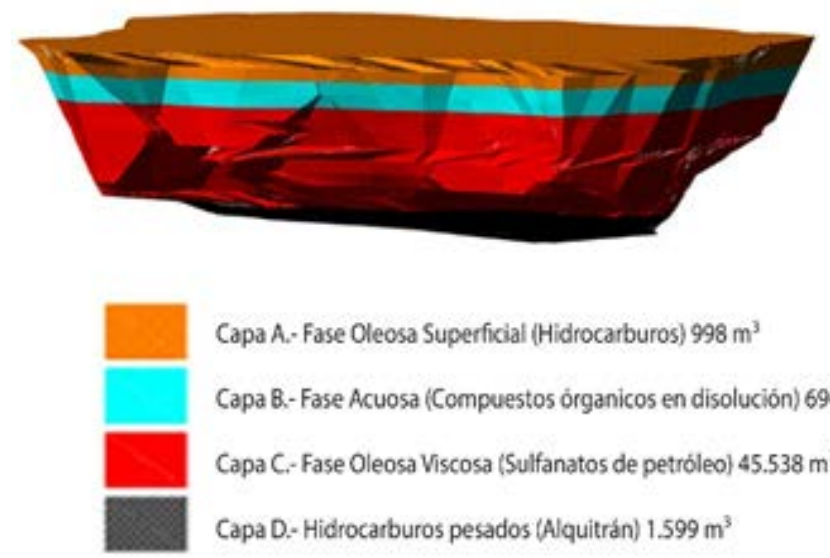

Capa A. Fase Oleosa Superficial (Hidrocarburos) $998 \mathrm{~m}^{3}$

Capa B. Fase Acuosa (Compuestos órganicos en disoluciön) $694 \mathrm{~m}^{3}$

Capa C.- Fase Oleosa Viscosa (Sulfanatos de petróleo) $45.538 \mathrm{~m}^{3}$

Capa D.- Hidrocarburos pesados (Alquitrán) $1.599 \mathrm{~m}^{3}$

Figura 5. Vista en 3D de las cuatro capas identificadas en la laguna principal y cubicación de los residuos presentes en cada capa. (TRAGSA and EMGRISA, 2013).

Figure 5. $3 D$ view of the four layers identified in the main lagoon and disposal of the residues. (TRAGSA and EMGRISA, 2013).

Se ha realizado una importante labor de caracterización de cada una de las capas de materiales depositados en la laguna principal. Para ello se tomaron muestras en 45 puntos de muestreo directo y se realizaron 31 vibrocores, de los que se recogieron 190 muestras del residuo y 14 muestras de la base.

Esta investigación permitió la caracterización exhaustiva de las distintas capas:

- Capa A. Es una fase oleosa superficial de menor densidad que flota sobre el conjunto. La capade color marrón oscuro se considera que está formada por hidrocarburos ligeros liberados de la masa de residuos. El espesor medio es de 9 $\mathrm{cm}$, con variaciones significativas entre puntos contiguos.

- Capa B. Es una fase acuosa, de color marrón y turbidez elevada. Aparentemente contiene abundantes compuestos orgánicos en disolución y suspensión, que originan su aspecto. EI espesor de esta capa presenta valores entre 2 y $12 \mathrm{~cm}$ (valor medio de $6 \mathrm{~cm}$ ). Su escaso volumen sugiere que se produce una filtración de los excedentes hacia el terreno circundante.

- Capa C. Es la más potente, formando el núcleo principal de la balsa. Se trata de una fase oleosa muy viscosa, de color negro. Su espesor varía en función de la profundidad en cada punto, alcanzando hasta $7 \mathrm{~m}$, con valores de entre 5 y 7 en la mayor parte de la balsa disminuyendo hacia las orillas exteriores. Esta capa está constituida por el residuo industrial vertido en el pasado (sulfonatos). La Fase 1A comprende la extracción del material de esta capa que pueda 
ser extraíble mediante bombeo, siendo objeto de la Fase 1B aquel que requiera medios mecánicos para su extracción.

- Capa D: Esta capa es la más densa y ocupa el fondo de la balsa. Es de color negro, está formada por hidrocarburos pesados, de viscosidad muy elevada (alquitranes) aunque con cierto grado de fluidez. Esta capa es bastante irregular, con puntos en los que se alcanza un metro de espesor y otros donde apenas tiene $5 \mathrm{~cm}$. La extracción de este material se realizará mediante medios mecánicos durante el desarrollo de las operaciones de la Fase 1B.

En la Tabla 1 se muestran los valores de los distintos parámetros físico-químicos analizados de las capas $C$ y D.

\begin{tabular}{llll}
\hline & Unidades & Capa C & Capa D \\
\hline Volumen & $\mathrm{m}^{3}$ & $45143^{*}$ & 4238 \\
Densidad & $\mathrm{kg} / \mathrm{m}^{3}$ & 1078.05 & 1379.8 \\
Peso & $\mathrm{t}$ & 48666 & 5848 \\
Viscosidad & $\mathrm{cP}$ & 38195.78 & 304872.29 \\
Agua & $\%$ & 28.28 & 7.1 \\
& $\mathrm{t}$ & 13853 & 157 \\
PC neto & $\mathrm{kcal} / \mathrm{kg}$ & 5610 & 3517 \\
Acidez & $\mathrm{mg} \mathrm{KOH} / \mathrm{g}$ & 33.2 & 25.7 \\
& $\mathrm{t} \mathrm{KOH}$ & 1626 & 57 \\
Azufre & $\%$ & 5.78 & 3.09 \\
& $\mathrm{t}$ & 2831.3 & 68.2 \\
Plomo & $\mathrm{mg} / \mathrm{kg}$ & 3873 & 4758 \\
& $\mathrm{t}$ & 189.7 & 10.5 \\
Zinc & $\mathrm{mg} / \mathrm{kg}$ & 997 & 440 \\
& $\mathrm{t}$ & 48.8 & 1.0 \\
PCBs & $\mathrm{mg} / \mathrm{kg}$ & 13.36 & 10.3 \\
& $\mathrm{~kg}$ & 627.0 & 22.7 \\
\hline
\end{tabular}

$(*)$ Incluve las canas A. B y C

Tabla 1. Características y concentraciones obtenidas en el estudio de caracterización de las capas C y D de la laguna principal (Mora Peris et al. 2017).

Table 1. Characteristics and concentrations obtained from the polluting material characterization study of the main lagoon (Mora Peris et al., 2017).

En la Figura 6 se muestra la disposición de una capa de limos situada en el fondo de la balsa de aproximadamente $0.5 \mathrm{~m}$ de espesor bajo las capas $\mathrm{C}$ y D. Esta capa de limos presenta una permeabilidad de $2 \times 10^{-7}$ $\mathrm{m} / \mathrm{s}$ (TRAGSA and EMGRISA 2013). En la Figura 6 se observa que como resultado de los trabajos previos al comienzo de la Fase $1 \mathrm{~A}$ se consiguió una caracterización geológica muy detallada del subsuelo. Un elemento esencial de cara a las actuaciones posibles para la protección de las aguas subterráneas en los trabajos futuros es la presencia de una capa infrayacente de limos con un espesor de $2 \mathrm{~m}$, situada entre las cotas 519 y $517 \mathrm{~m} \mathrm{s.n.m}$. y una permeabilidad de $2.5 \times 10^{-9} \mathrm{~m} / \mathrm{s}$.

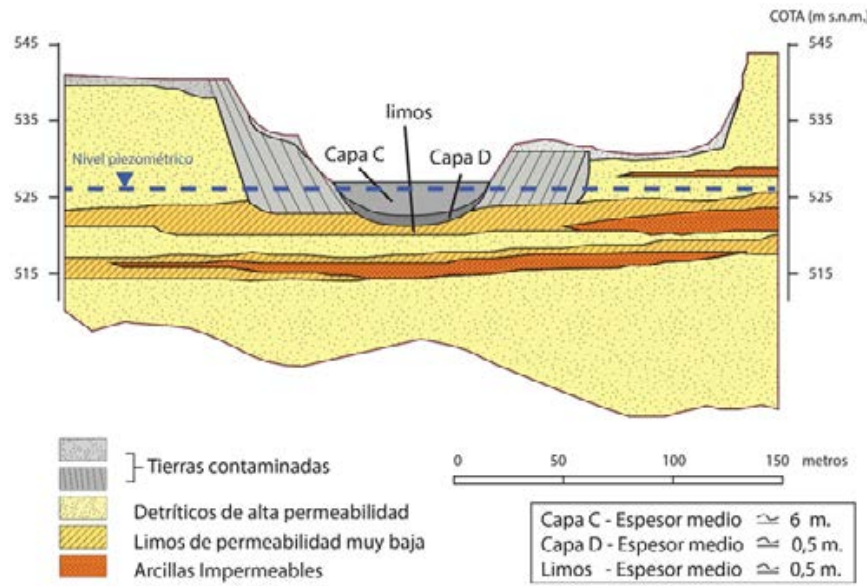

Figura 6. Corte geológico del subsuelo de la parcela.

Figure 6. Subsoil geological section of the plot.

\section{Marco hidrológico e hidrogeológico de la zona}

\section{Hidrología}

La laguna se encuentra enclavada dentro del Parque Regional del Sureste que se estructura en torno a los ejes de los cursos bajos de los ríos Manzanares y Jarama, constituyéndose sus cauces como elementos físicos determinantes en la configuración de este espacio. Las actividades extractivas en la vega del Jarama han ido ocupando paulatinamente el espacio agrícola y dejando su huella en el paisaje en forma de lagunas de distintas formas y tamaños, ligadas principalmente a la cuenca del río. Se ha inventariado la presencia de 123 humedales en este espacio natural protegido donde estas lagunas son hoy el hábitat predilecto de numerosas especies animales, destacando especialmente las aves. La valoración ambiental de estos ecosistemas, atendiendo a criterios biológicos, ecológicos y antrópicos, es alta en casi uno de cada cuatro humedales (Parque Regional del Sureste. Consejería de Medio Ambiente y Ordenación delTerritorio de la Comunidad de Madrid, 31/05/17, http://www.parqueregionalsureste.org).

Con el fin de disponer de información de partida correspondiente a distintos puntos de masas de aguas, en primer lugar, se procedió a recabar información sobre puntos de control de calidad de agua superficial próximos que pudieran ser controlados de forma sistemática por el organismo competente, esto es, la Confederación Hidrográfica del Tajo (CH Tajo), así como de estudios previos al actual que pudieran haber sido realizados en un entorno lagunar, más o menos próximo.

a. Punto de control de la $\mathrm{CHTajo}$

Dentro de la Red CEMAS de la CHTajo, a escasos 4 $\mathrm{km}$ al oeste de la laguna de estudio, se encuentra la estación número $50 \mathrm{E}$. Presa del Rey - Jarama con códigoTA58205002 localizada en el cauce del río Jarama (Figura 7). 


\section{b. Laguna de Las Madres}

La Laguna de las Madres esta próxima a la parcela de estudio (Figura 7), tiene un origen similar, en cuanto a su génesis por actividades mineras y por proximidad a la zona de estudio. En esta laguna el grupo de investigación del agua del Consejo Superior de Investigaciones Científicas (CSIC) lleva a cabo estudios limnológicos desde 1991 (Ecología acuática. Consejo Superior de Investigaciones Científicas (CSIC), 31/05/2017, http://www.humedalesibericos.com), realizando un seguimiento mensual desde septiembre de dicho año, en el que se miden diversos parámetros físico químicos de interés para la conservación y evaluación de calidad de las aguas.

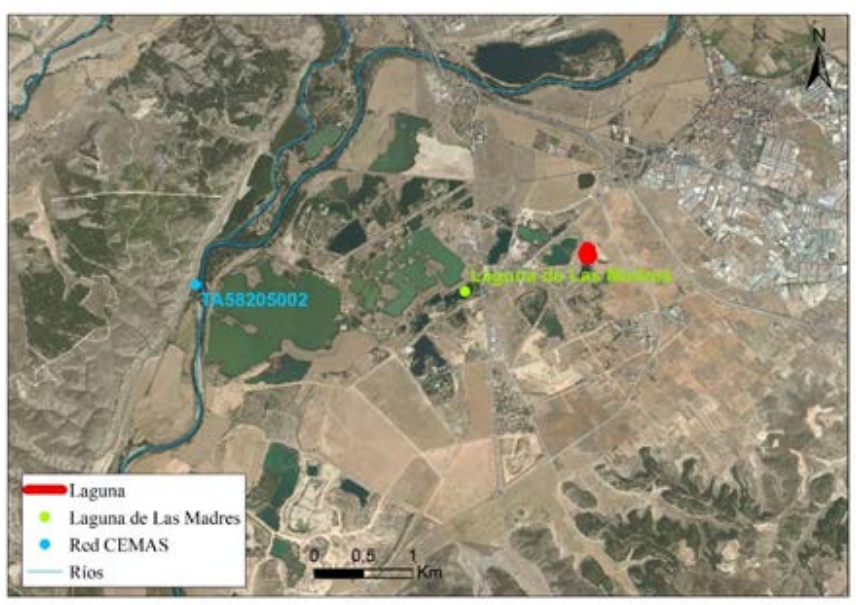

Figura 7. Situación del punto de la Red CEMASTA58205002 E. Presa del Rey - Jarama y de la Laguna de las Madres en el entorno de la laguna de estudio.

Figure 7. Location of the CEMAS TA58205002 Network point E. Presa del Rey - Jarama and the Lagoon of "Las Madres", in the surroundings of the study lagoon.

\section{c. Lagunas situadas inmediatamente próximas a la} parcela de estudio

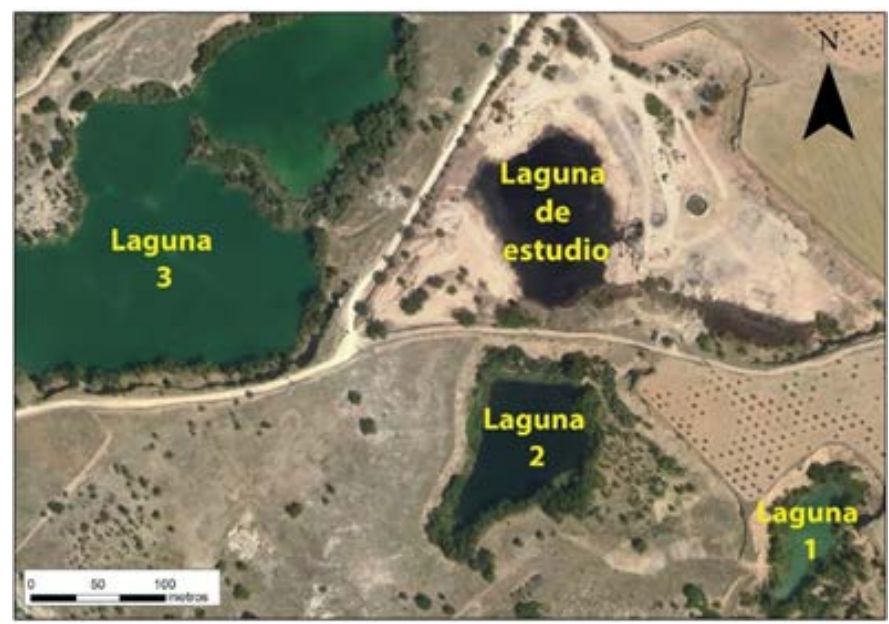

Figura 8. Situación de las lagunas anexas a la parcela de estudio. Figure 8. Situation of the lagoons attached to the study plot.
Al comienzo de los trabajos de TRAGSA y CGSi, en mayo de 2017, en el marco de los estudios que se recogen en este artículo se realizó un reconocimiento en detalle de la zona sobre las lagunas próximas a la balsa, cuya situación se presenta en la Figura 8.

Los resultados del análisis in situ realizado el 24 de mayo del 2017 de los parámetros físico-químicos (Conductividad eléctrica, $\mathrm{pH}$ y temperatura) se recogen en la Tabla 2. No se han detectado en esta campaña caracteres organolépticos (color, olor) que pudieran indicar presencia de contaminación en las aguas por hidrocarburos.

\begin{tabular}{cccc}
\hline Laguna Muestreada & Conductividad $(\mu \mathrm{S} / \mathrm{cm})$ & $\mathrm{pH}$ & Te mperatura $\left({ }^{\circ} \mathrm{C}\right)$ \\
\hline $\mathrm{N} \cdot{ }^{\circ} 1$ & 1310 & 8.2 & 26.2 \\
$\mathrm{~N} \cdot{ }^{\circ} 2$ & 2020 & 8.3 & 26.7 \\
$\mathrm{~N} .{ }^{\circ} 3$ & 1590 & 8.5 & 27.3 \\
\hline
\end{tabular}

Tabla 2. Parámetros in situ medidos en las lagunas próximas a la balsa de estudio (TRAGSA and CGSi, 2017 a).

Table 2. On-site parameters measured in the lagoons near the study raft (TRAGSA and CGSi, 2017 a).

Estos valores recogidos "in situ" en las lagunas adyacentes a la balsa se encuentran dentro de los intervalos de variación de los registrados históricamente en la laguna de Las Madres: conductividad $1000-$ $3500 \mu \mathrm{S} / \mathrm{cm}$; $\mathrm{pH} 6-8.5$; y temperatura $20-28{ }^{\circ} \mathrm{C}$. (Ecología acuática. Consejo Superior de Investigaciones Científicas (CSIC), 31/05/2017, http://www.humedalesibericos.com).

Como en el caso de la Laguna de La Madres, las tres masas de agua conformadas por estas tres lagunas tienen una relación hidráulica inmediata con las aguas subterráneas, cuya calidad es necesario controlar y preservar en cualquier actuación que se realice en los trabajos de restauración ambiental de la balsa. También estas tres lagunas están relacionadas con huecos mineros (gaveras) y carecen de alimentación hídrica con emisarios superficiales, siendo su principal aporte de origen subterráneo, constituyendo lo que, en términos hidrogeológicos se denomina humedal hipogénico.

El interés del control de estas tres lagunas, además del mantenimiento medioambiental del correspondiente ecosistema, radica en que la medición sistemática de su estado constituye una herramienta de control de las actividades de restauración que se realicen en la balsa, debido a su conexión hidráulica directa con las aguas subterráneas. Esta conexión también condiciona, desde el punto de vista hidrodinámico, que cualquier bombeo próximo a la balsa pueda verse afectado por un flujo importante de agua procedente de estas lagunas hacia dicho pozo debido a que conforman el mismo acuífero.

\section{Hidrogeología}

Desde el punto de vista hidrogeológico, la balsa de Arganda (Madrid) se localiza en la Masa de Agua Sub- 
terránea (MASb) 030.007 Aluviales: Jarama - Tajuña (Figura 9), dentro de la Demarcación Hidrográfica del Tajo (Centro de Descargas de Infraestructura de Datos Espaciales (IDE). Ministerio para la Transición Ecológica, 04/05/17, https://www.miteco.gob.es/es/ cartografia-y-sig/ide/descargas/agua/masas-de-aguaphc-2015-2021.aspx). La MASb está formada por los depósitos aluviales y de llanura de inundación de los ríos Jarama y una parte del Tajuña, junto con depósitos de terraza aluvial. Incluye gravas poligénicas, arenas, limos y arcillas del Cuaternario. Los rellenos aluviales de ambos ríos discurren sobre facies evaporíticas delTerciario. La zona no saturada está formada por gravas poligénicas, arenas, limos y arcillas del Cuaternario.

El acuífero donde está situada la balsa (MASb 030.007 Aluviales: Jarama - Tajuña) tiene carácter de acuífero libre con porosidad primaria intergranular y por tanto con gran vulnerabilidad a la contaminación.

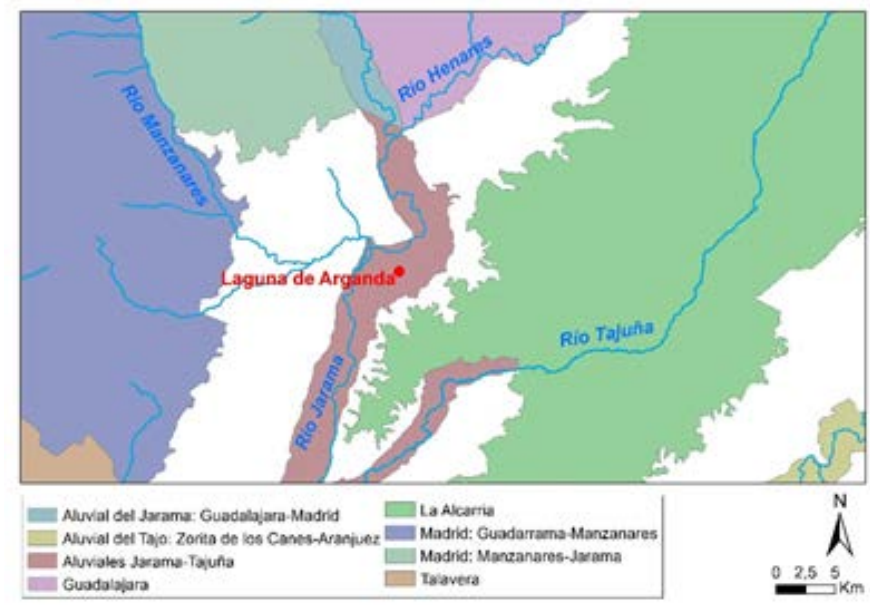

Figura 9. Masas de agua subterráneas en el entorno de la zona de estudio (Elaboración propia con bases de Masas de agua subterránea PHC (2015-2021), Centro de Descargas de Infraestructura de Datos Espaciales (IDE). Ministerio para la Transición Ecológica, 04/05/17).

Figure 9. Bodies of underground water in the surroundings of the study area lown elaboration with PHC underground waterbody base (2015-2021), Spatial Data Infrastructure Download Center. Ministry for Ecological Transition, 04/05/17).

Para tener un mejor conocimiento de la hidrogeología de la zona se ha procedido a consultar diversas bases de datos de puntos de agua. 1) la base de puntos de agua del IGME, 2) MAPAMA y 3) la de la CHTajo. En la Figura 10 se representan los puntos obtenidos en dichas consultas.

De entre todos los puntos recopilados el que presenta un mayor interés es el punto de control de la CHTajo denominado 0701 TAIG000595, pues al ser un punto controlado sistemáticamente por la Administración Hídrica dispone de una serie histórica extensa a efectos comparativos durante las operaciones de recuperación de la balsa de Arganda.

El punto 07.01-TAIG000595 servirá de punto de re- ferencia de calidad de agua para las actuaciones que puedan realizarse en la balsa. Este punto dispone de un gran número de mediciones de análisis químicos (Visor CHTajo. Confederación Hidrográfica del Tajo (CHTajo), 31/05/17, http://www.chtajo.es/LaCuenca/Paginas/visor.aspx).

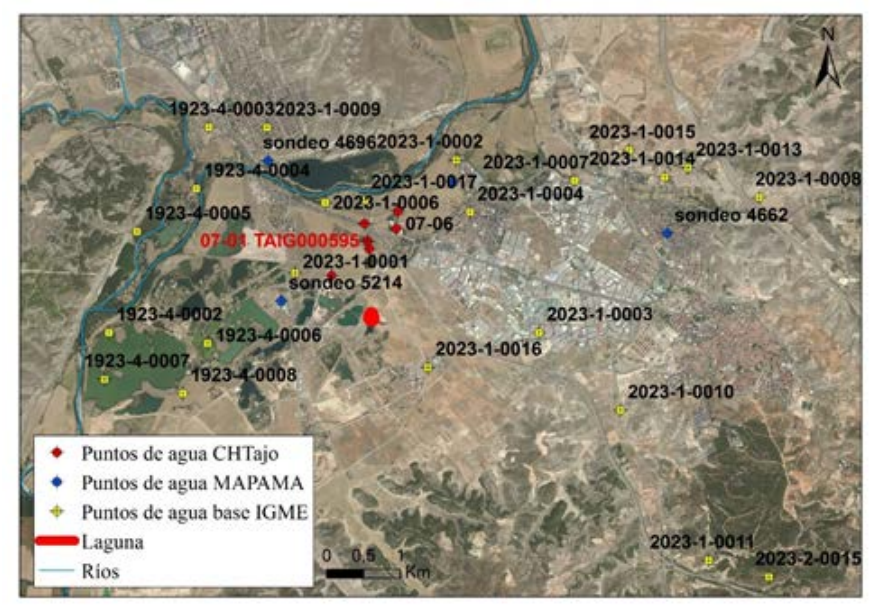

Figura 10. Puntos de agua obtenidos en la consulta a diversos organismos.

Figure 10. Water points obtained in consultation with several organisms.

\section{Metodología y trabajos realizados}

Los trabajos de control hidrogeológico fueron realizados en 2017, de forma previa al comienzo de la Fase $1 \mathrm{~B}$, con objeto de poder planificar dicha fase con suficiente antelación. El alcance de los mismos era el necesario para disponer del conocimiento hidrogeológico de la zona que permitiera una identificación de las alternativas de control y gestión del agua subterránea durante las operaciones de la Fase 1B, y la selección de la considerada como más adecuada.

Los trabajos incluyeron las siguientes actividades:

- Definición del flujo subterráneo en la parcela.

- Caracterización de la calidad de las aguas en el entorno de la parcela.

- Realización e interpretación de ensayos de bombeo.

- Modelización con MODFLOW para cuantificación de los flujos de agua subterránea.

- Identificación, caracterización y evaluación de alternativas.

A continuación, se describen de forma sintética cada una de estas.

\section{Definición del flujo subterráneo en la parcela}

A lo largo del desarrollo de los distintos estudios (TRAGSA and EMGRISA, 2013) se ha llevado a cabo una completa investigación del subsuelo y las aguas subterráneas de la parcela, a partir de la ejecución 
de un gran número de sondeos y piezómetros cuya situación se muestra en la Figura 11. A partir de las mediciones del nivel piezométrico y de la recogida de muestras de agua subterránea en estos puntos ha podido establecerse la red de flujo de las aguas subterráneas y conocer su calidad. La determinación de la piezometría de la parcela de estudio es fundamental con el fin de establecer el esquema conceptual de flujo de las aguas subterráneas.

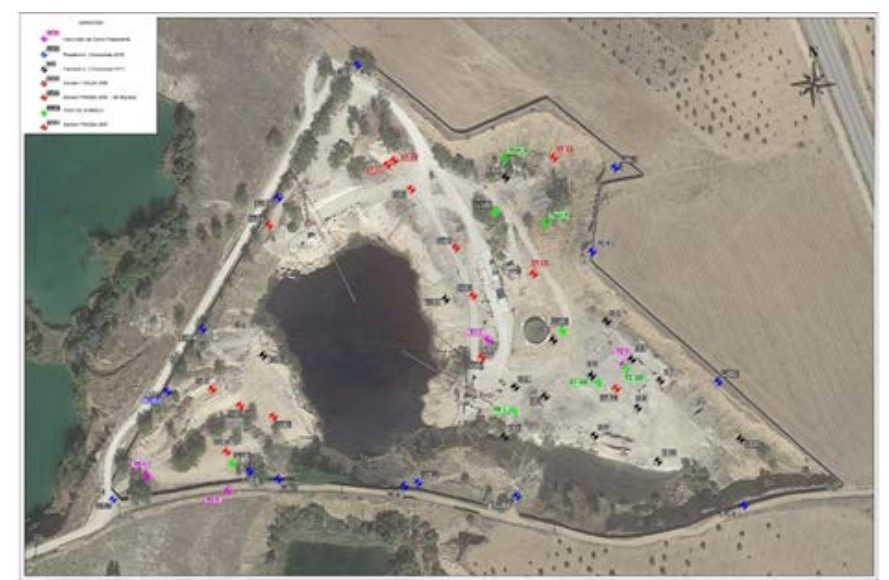

Figura 11. Sondeos de investigación y control realizados en la parcela.

Figure 11. Research and control drillings constructed on the plot.

Se han realizado tres campañas de piezometría durante los meses de Mayo, Junio y Julio de 2017 (Figura 12), lo que ha permitido establecer las direcciones y sentido de flujo principales que serán previsiblemente similares a los que se darán durante las labores de extracción de los residuos de la Fase 1B a realizar en el periodo seco. Los resultados de estas piezometrías son coherentes con las realizadas en todos los estudios previos (TRAGSA and EMGRISA, 2013). En estas campañas de 2017 se han tomado como puntos de control los piezómetros correspondientes a la red de control perimetral ya existente con los sondeos de la serie PC-1 a PC-10.

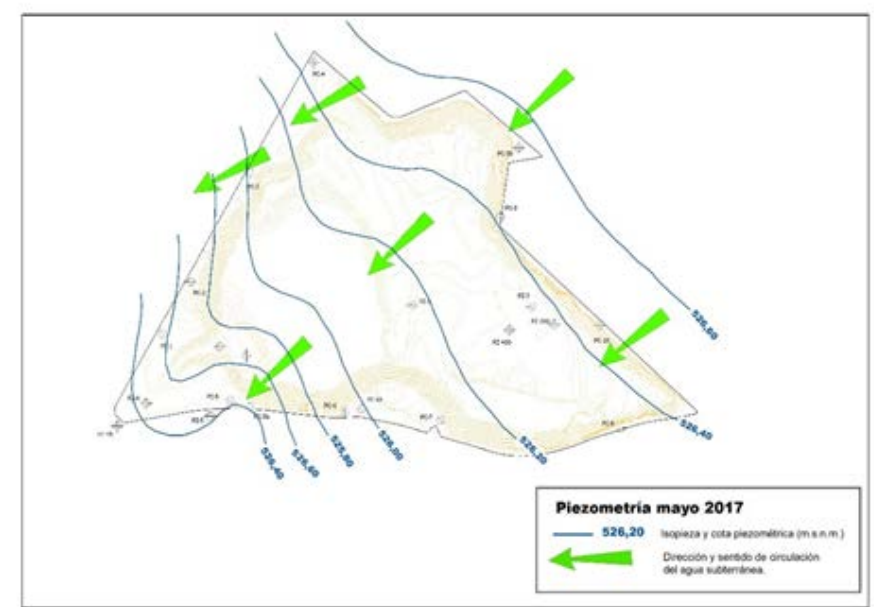

Figura 12a. Mapa de isopiezas en mayo 2017.

Figure 12a. Piezometric map May 2017.
A partir de la interpretación de los datos obtenidos se ha podido establecer una piezometría y se han identificado las zonas de descarga del acuífero que pueden ser afectadas por la circulación de las aguas subterráneas (TRAGSA and CGSi, 2017a).

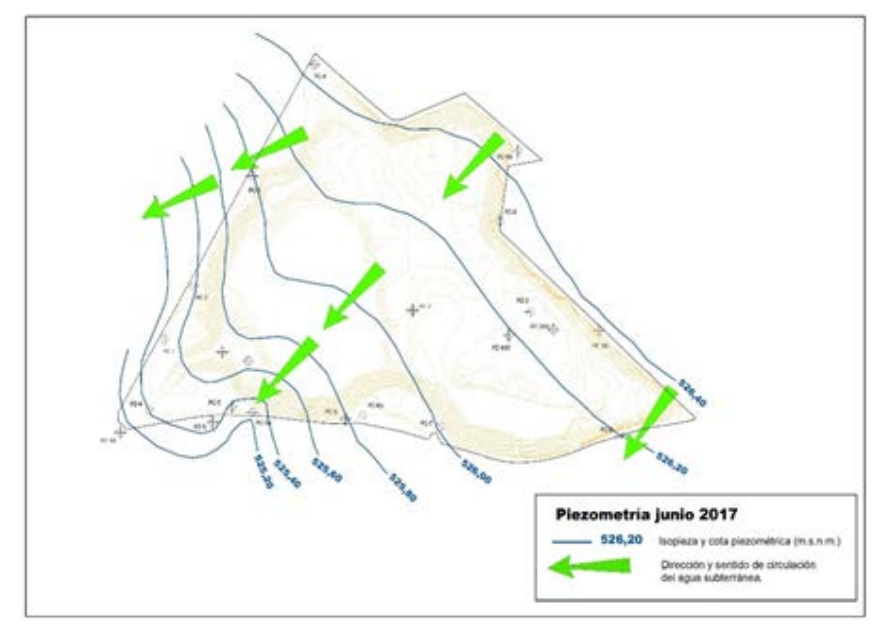

Figura 12b. Mapa de isopiezas en junio 2017.

Figure 12b. Piezometric map June 2017.

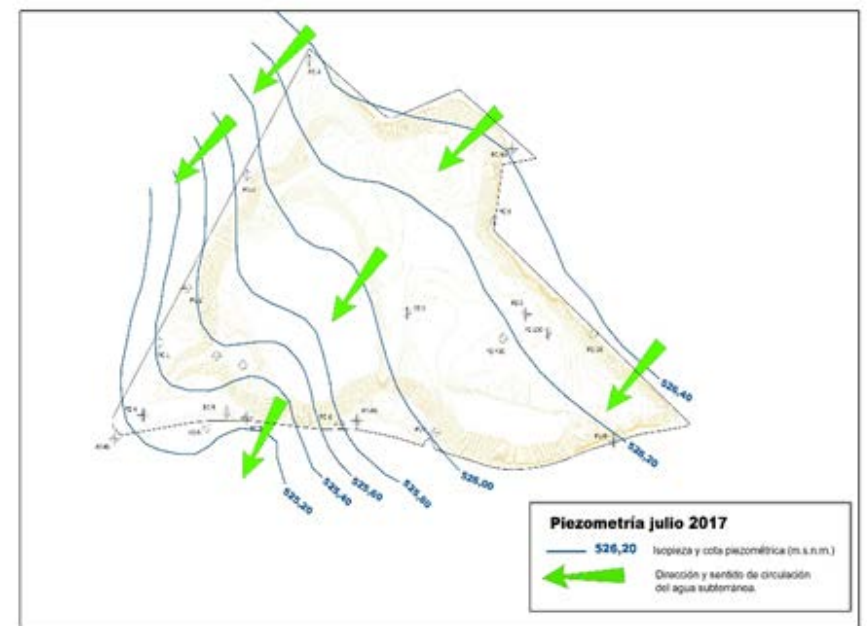

Figura 12c. Mapa de isopiezas en julio 2017.

Figure 12c. Piezometric map July 2017.

Existe una relación hidráulica directa entre el flujo de las aguas subterráneas y las tres lagunas adyacentes donde se produce la descarga de las mismas, dado el carácter de humedal hipogénico de estas masas de agua superficial. Esta característica también determina la morfología de la superficie piezométrica.

Se obtienen las siguientes conclusiones sobre las características del flujo subterráneo en la parcela:

- El flujo de tiene una componente general NE-SO, con sentido hacia el SO. Se observa una descarga principal hacia la masa de agua situada al sur y también, pero en menor medida, hacia la laguna situada al oeste y se apunta también un probable flujo hacia la laguna SE exterior. 
- El gradiente hidráulico es del orden del $0.44 \%$, lo que corresponde a un medio poroso (acuífero) bastante permeable, en su conjunto.

- La cota media piezométrica en el entorno de la balsa es de $526 \mathrm{~m}$ s.n.m. Puesto que el muro se sitúa a cota aproximada $522 \mathrm{~m}$ s.n.m., lo esperable es que el agua en esa cota se encuentre a una presión de unos $4 \mathrm{~m}$ de columna de agua.

- Se pone claramente de manifiesto que la morfología de la superficie piezométrica corresponde a una zona de humedal hipogénico, dada la proximidad a las tres masas de aguas superficiales.

\section{Caracterización de la calidad de las aguas en el entor- no de la parcela}

En junio de 2017 se tomaron muestras de agua superficiales, para un análisis químico muy exhaustivo en los laboratorios de LABAQUA, S.A, con el fin de conocer la calidad de las aguas y la posible afección en las lagunas situadas en el exterior de la parcela, que constituyen las zonas de descarga del flujo subterráneo de acuerdo con su característica de humedal hipogénico.

En ninguna de las tres lagunas limítrofes con la parcela estudiada se superan los valores establecidos en el Real Decreto 817/2015, de 11 de septiembre, por el que se establecen los criterios de seguimiento y evaluación del estado de las aguas superficiales y las normas de calidad ambiental (Tabla 3).

Como consecuencia de lo anterior se puede concluir que la afección sobre el medio hídrico no ha atravesado los límites de la parcela donde se ubica la balsa.

\section{Realización e interpretación de ensayos de bombeo}

Una vez conocido el modelo hidrogeológico de funcionamiento conceptual, era preciso abordar la fase de evaluación cuantitativa de los parámetros hidrodinámicos para poder analizar y discutir las posibles alternativas de drenaje del subsuelo de la balsa durante las operaciones de extracción de los residuos.

La realización y posterior interpretación de ensayos de bombeo es el método con mayor garantía para co-

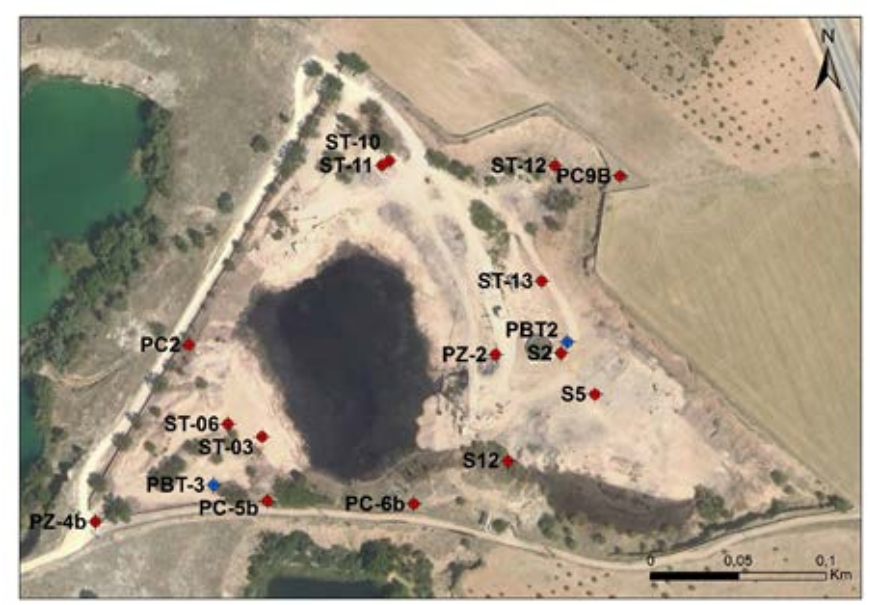

Figura 13. Situación de los puntos de bombeo y control en los ensayos de bombeo realizados (pozos de bombeo en azul y piezómetros de observación en rojo).

Figure 13. Situation of the pumping and control points in the pumping tests carried out (pumping wells in blue and observation piezometers in red).

\begin{tabular}{lccccc}
\hline Parámetros & Unidades & R.D. 817/2015 & $\begin{array}{c}\text { LAGUNA S } \\
\mathbf{2 0 / 0 6 / 2 0 1 7}\end{array}$ & $\begin{array}{c}\text { LAGUNA SE } \\
\mathbf{2 0 / 0 6 / 2 0 1 7}\end{array}$ & $\begin{array}{c}\text { LAGUNA W } \\
\mathbf{2 0 / 0 6 / 2 0 1 7}\end{array}$ \\
\hline PAH & & & & & \\
\hline Naftaleno & $\mu \mathrm{g} / 1$ & 2.4 & $<0.5$ & $<0.5$ & $<0.5$ \\
Antraceno & $\mu \mathrm{g} / \mathrm{l}$ & 0.1 & $<0.01$ & $<0.01$ & $<0.01$ \\
Benzo(b) fluoranteno & $\mu \mathrm{g} / 1$ & 0.03 & $<0.01$ & $<0.01$ & $<0.01$ \\
Benzo(k) fluoranteno & $\mu \mathrm{g} / 1$ & 0.03 & $<0.01$ & $<0.01$ & $<0.01$ \\
Benzo(a) pireno & $\mu \mathrm{g} / 1$ & 0.05 & $<0.005$ & $<0.005$ & $<0.005$ \\
Indeno (1,2,3-c,d) & $\mu \mathrm{g} / 1$ & & $<0.01$ & & \\
pireno & $\mu \mathrm{g} / 1$ & 0.002 & $<0.01$ & $<0.01$ & $<0.01$ \\
Benzo(g,h,i) perileno & & & & & $<0.01$ \\
Metales & $\mu \mathrm{g} / 1$ & 50 & 5 & 4 & 6 \\
\hline Arsénico & $\mu \mathrm{g} / 1$ & $<0.08-0.25$ & $<1$ & $<1$ & $<1$ \\
Cadmio & $\mu \mathrm{g} / 1$ & 50 & $<2$ & 3 & $<2$ \\
Cromo & $\mu \mathrm{g} / 1$ & $5-120$ & $<2$ & $<2$ & $<2$ \\
Cobre & $\mu \mathrm{g} / 1$ & 7.2 & $<20$ & $<20$ & $<2$ \\
Plomo & $\mu \mathrm{g} / 1$ & 20 & $<2$ & & $<20$ \\
Níquel & $\mu \mathrm{g} / 1$ & 1 & $<2$ & $<2$ & \\
Selenio & $\mu \mathrm{g} / 1$ & $30-500$ & 3 & 6 & $<2$ \\
Zinc & & & & & \\
\hline
\end{tabular}

Tabla 3. Resultados analíticos obtenidos en las lagunas circundantes y comparación con la normativa aplicable.

Table 3. Analytical results from the surrounding lagoons and comparison with the applicable legislation. 
nocer las características hidráulicas de los acuíferos y de la calidad constructiva de las captaciones.

Los ensayos de bombeo se han llevado a cabo entre el 4 y el 11 de septiembre de 2017 en los sondeos PBT-2 y PBT-3 (Figura 13) y en cada ensayo se han controlado los niveles piezométricos, tanto en el pozo de bombeo como en distintos piezómetros del entorno para el control de la evolución espaciotemporal de los niveles.

Para la interpretación de los ensayos y las recuperaciones correspondientes (Figura 14) se han utilizado desarrollos informáticos propios que consideran la circulación del agua subterránea en un pozo a partir de la Ley de Darcy y la ecuación de la conservación de la masa en condiciones ideales y plantean la resolución analítica mediante el método de Theis y la simplificación de Jacob. Se han obtenido valores de los parámetros muy similares y coherentes entre sí.

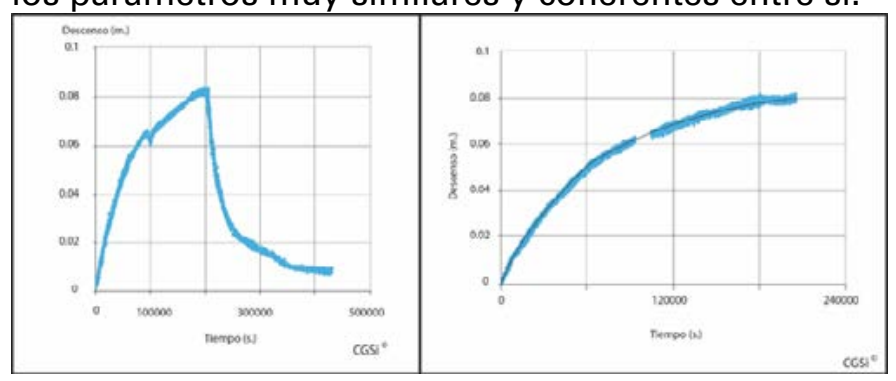

Figura 14. Gráfico de evolución de los niveles piezométricos en PZ$4 \mathrm{~b}$ durante el ensayo de bombeo del sondeo PBT-3 y ajuste para la interpretación por el método deTheis.

Figure 14. Chart of evolution of the piezometric levels in PZ-4b during the PBT-3 pumping test and Theis interpretation.

Al tratarse de un medio heterogéneo y anisótropo, lo importante es obtener valores medios que permitan tratar el medio como homogéneo e isótropo a escala global a los efectos de la modelización (Tabla 4).

En la tabla 5 se muestran los resultados obtenidos en los ensayos realizados, tanto en 2017 como en 2013 (TRAGSA and EMGRISA 2013), y la coherencia que se obtiene en los mismos en cuanto a los valores medios de transmisividad y permeabilidad considerando un espesor del acuífero de $30 \mathrm{~m}$. Estos valo- res corresponden a un medio muy transmisivo. De acuerdo con los valores obtenidos de coeficiente de almacenamiento el acuífero es libre con un alto grado de interconexión, si bien se han detectado zonas con carácter de semiconfinamiento, debido posiblemente a ciertos entornos litológicos con niveles más impermeables por su contenido en limos y arcillas.

También se ha realizado una estimación de los radios de influencia en los bombeos (Figura 15), obteniendo unos valores entre $150-300 \mathrm{~m}$.

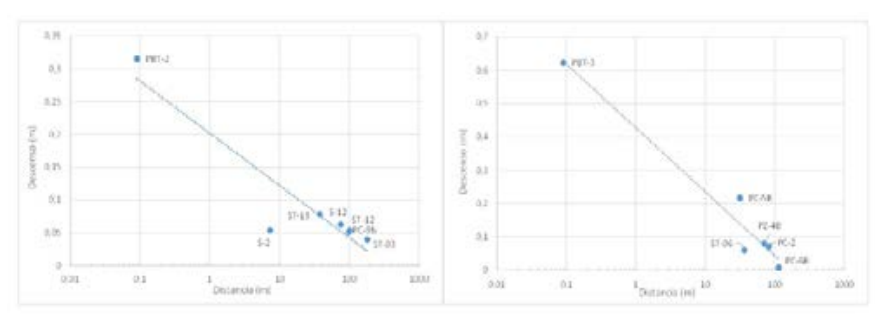

Figura 15. Ajuste de descensos para la estimación gráfica de los radios de influencia.

Figure 15. Adjustment of decreases for the graphic estimation of the influence radius.

\section{Modelización con MODFLOW para cuantificación del flujo subterráneo}

Una vez conocido tanto el modelo hidrogeológico conceptual de funcionamiento del acuífero, como los valores medios de los parámetros hidrodinámicos, es posible realizar la simulación numérica considerando distintas hipótesis de drenaje de las aguas subterráneas, así como los correspondientes caudales y volúmenes de agua a extraer.

Entre los modelos que habitualmente se utilizan para cuantificar el flujo subterráneo es de uso habitual en ingeniería por su flexibilidad y eficacia en medios porosos el modelo MODFLOW (USGS, 2016), empleado para estudiar los supuestos que aquí se presentan (TRAGSA and CGSi, 2017 b).

MODFLOW es un código para la modelización del flujo de aguas subterráneas basado en diferencias finitas de-

\begin{tabular}{cccccc}
\hline \multicolumn{2}{c}{ Media PBT-3 } & \multicolumn{2}{c}{ Media PBT-2 } & \multicolumn{2}{c}{ Promedio PBT-2 - PBT-3 } \\
\hline $\mathrm{T}\left(\mathrm{m}^{2} /\right.$ dia $)$ & $\mathrm{K}(\mathrm{m} / \mathrm{d})$ & $\mathrm{T}\left(\mathrm{m}^{2} /\right.$ dia $)$ & $\mathrm{K}(\mathrm{m} / \mathrm{d})$ & $\mathrm{T}\left(\mathrm{m}^{2} /\right.$ dia $)$ & $\mathrm{K}(\mathrm{m} / \mathrm{d})$ \\
\hline 1002.17 & 33.41 & 1655.96 & 55.20 & 1329.06 & 44.30 \\
\hline
\end{tabular}

Tabla 4. Valores medios de los parámetros hidrodinámicos obtenidos en los aforos.

Table 4. Average values of the hydrodynamic parameters obtained.

\begin{tabular}{ccc}
\hline Campaña de aforos & Transmisividad $\left(\mathbf{m}^{\mathbf{2}} / \mathbf{d i ́ a}\right)$ & Permeabilidad (m/día) \\
\hline TRAGSA-EMGRISA-2013 & 1623.33 & 49.74 \\
\hline TRAGSA-CGSi-2017 & 1329.06 & 44.3 \\
\hline
\end{tabular}

Tabla 5. Valores medios de transmisividad y permeabilidad obtenidos en las campañas realizadas.

Table 5. Average values of transmission and permeability obtained in the campaigns carried out. 
sarrollado por el Servicio Geológico de Estados Unidos (USGS). MODFLOW simula en régimen estacionario y transitorio un sistema acuífero irregular que puede ser confinado, no confinado, o mixto. Tuvo su aparición en 1984 y ha tenido un constante desarrollo hasta la actualidad. Las capacidades de MODFLOW permiten representar el flujo regional o local de agua subterránea y su interacción con los cuerpos de agua superficial.

Hay que tener siempre en cuenta que los modelos de simulación son aproximaciones a la realidad, pero no son la realidad misma, y por lo tanto todos los resultados que se obtengan han de ser verificados posteriormente con la experiencia práctica y la empírica.

La modelización con MODFLOW ha sido realizada en colaboración con la Sección de Hidrogeología y Geología Aplicada (HYGA) del Grupo de Ingeniería del Agua y del Medio Ambiente (GEAMA) de la Universidad de La Coruña.

El objetivo fundamental de la simulación realizada con MODFLOW es obtener datos cuantitativos que permitan efectuar un análisis de alternativas de gestión de las aguas subterráneas durante las operaciones de la Fase 1B. La modelización se ha realizado en régimen estacionario, en flujo bidimensional, considerando un medio homogéneo e isótropo.

a. Modelo conceptual

El modelo base ha consistido en simular, en condiciones estacionarias, el flujo en un único acuífero no confinado isótropo cuya base se encuentra a la cota 493 m s.n.m. Las condiciones de contorno impuestas garantizan que la dirección de las líneas de corriente, principalmente, se produzcan desde el Noreste hacia el Suroeste (Figura 16).

En el modelo se han tenido en cuenta las lagunas existentes al Oeste, Sur y Este de la balsa de residuos, y cuyas distancias a las mismas oscilan entre los 57 y $210 \mathrm{~m}$, siendo la laguna más cercana la laguna Sur. Para simular las distintas lagunas, se ha supuesto que el volumen de terreno que ocupan en el modelo tiene una permeabilidad muy alta, con el fin de que el nivel se uniformice inmediatamente, en caso de que se vea

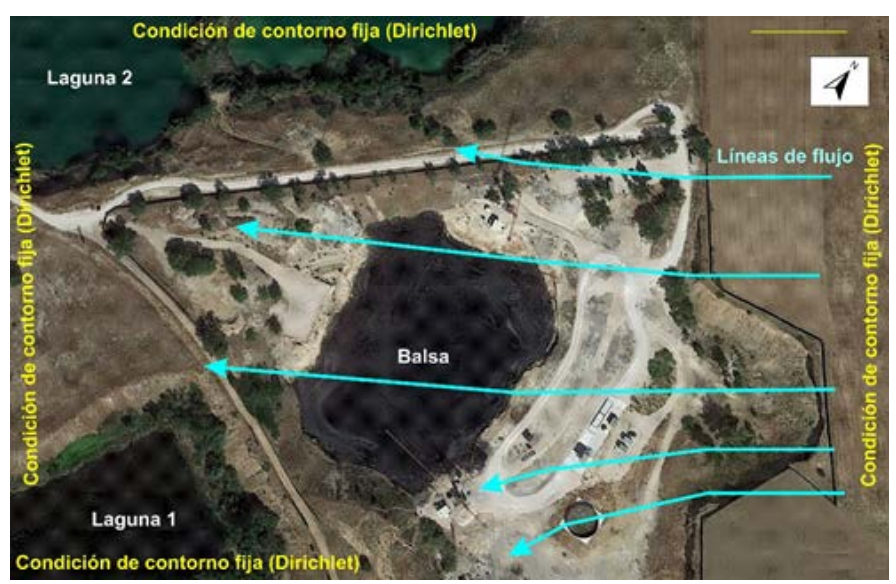

Figura 16. Modelo conceptual (Imagen Google).

Figure 16. Conceptual model (Google image). afectada alguna de ellas por el radio de influencia generado por los bombeos en los pozos que se simulen en los modelos.

Desde el punto de vista vertical, se han tenido en cuenta dos materiales: un material acuífero y el sustrato impermeable. No se ha considerado para la modelización efecto de recarga por lluvia, dada la limitada superficie considerada del dominio y la previsión de realizar las operaciones en época de estiaje.

b. Modelo numérico. Escenarios

Se ha construido una malla de 5220 elementos/capa considerándose los distintos materiales considerados en la modelización (Figura 17).

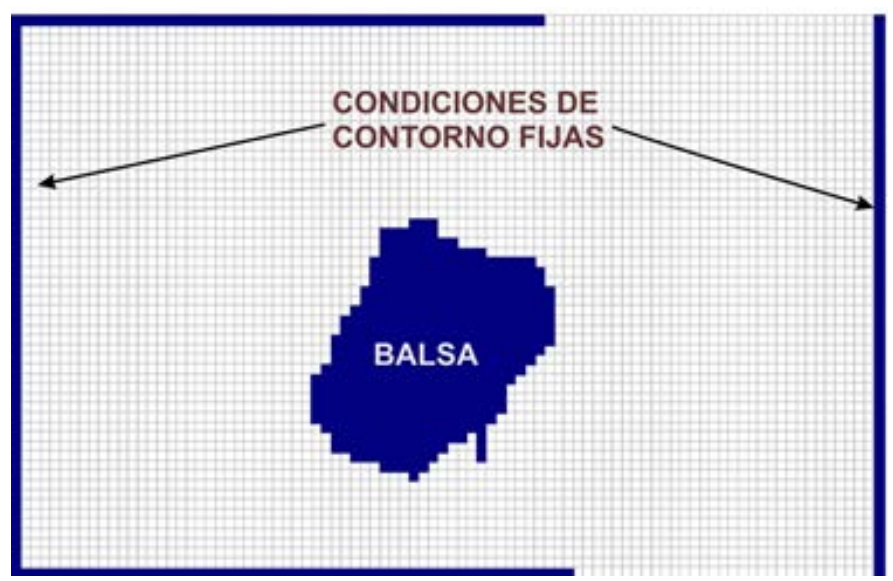

Figura 17. Características consideradas en la modelización numérica con MODFLOW.

Figure 17. Characteristics considered in numerical modelling with MODFLOW.

La permeabilidad considerada para el acuífero ha sido la obtenida en los ensayos de bombeo de $5.1 \mathrm{x}$ $10^{-4} \mathrm{~m} / \mathrm{s}$, y la de las lagunas en la zona del dominio del modelo de $100 \mathrm{~m} / \mathrm{s}$.

La calibración del modelo ha consistido en identificar adecuadamente las distintas condiciones de contorno, con el fin de obtener una representación de las isopiezas y líneas de flujo similares a las medidas en las distintas campañas de medición de piezometría: campañas de 2009, 2010, 2011, 2013, 2014 y 2017 (TRAGSA and EMGRISA, 2013 y TRAGSA and CGSi, 2017a).

Se han simulado tres escenarios distintos en función de la geología de la zona. En la Figura 18 se muestran los tres escenarios de comportamiento hidrogeológico del medio considerados en la modelización. EI primer escenario considera un único acuífero. En el segundo escenario se ha considerado que la capa de arcilla de $2 \mathrm{~m}$ de espesor, detectada en el subsuelo, en el entorno de la balsa, pueda tener una extensión que delimite y aísle dos acuíferos de tal forma que la laguna sólo se encuentre afectada por el acuífero superior (TRAGSA and EMGRISA, 2013). También se ha considerado un tercer escenario de desconexión por los limos detectados en el fondo de la balsa, suponiendo la continuidad lateral de los mismos. 


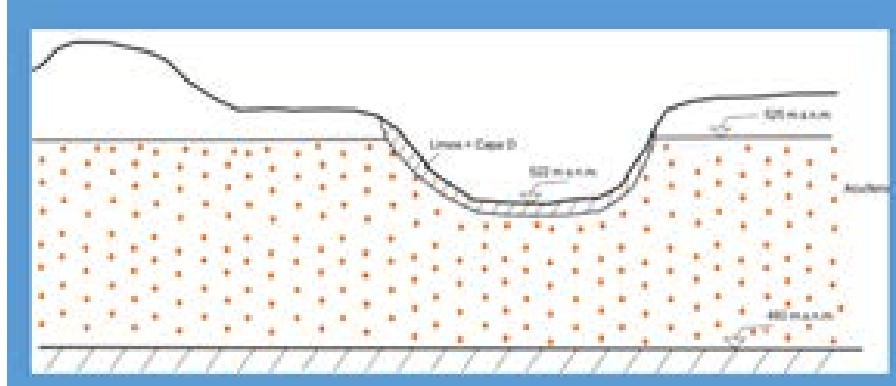

a) Acuifero interconectado

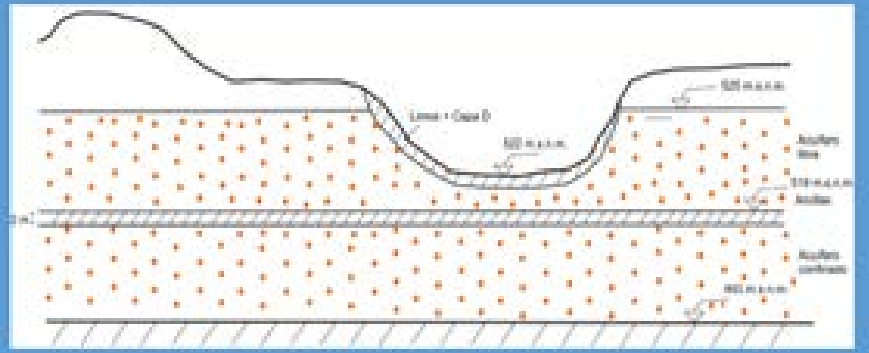

b) Acuifero superior desconectado en las arcilla

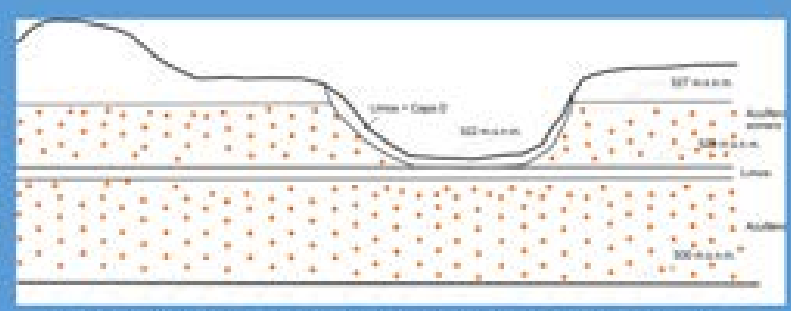

c) Acuifero superior desconectado en los limos

Figura 18. Hipótesis de funcionamiento hidrogeológico.

Figure 18. Hydrogeological functioning hypothesis.

Como alternativas de drenaje a simular se han supuesto las siguientes condiciones:

- Drenaje lineal mediante batería de 12 pozos.

- Drenaje lineal mediante zanja de $120 \mathrm{~m}$.

- Sistema de drenaje perimetral con anillo de 43 pozos.

- Sistema de drenaje perimetral con zanja.

Las hipótesis de bombeo han contemplado en primer lugar producir descensos piezométricos hasta alcanzar la cota de $522 \mathrm{~m}$ s.n.m., correspondiente al fondo de la balsa. De forma complementaria se han realizado simulaciones hasta alcanzar las cotas de 523 m s.n.m y 524 m s.n.m., para poder considerar los supuestos de que el fondo de la balsa sea irregular y que los espesores del residuo depositado sean inferiores a lo previsto.

\section{Identificación, caracterización y evaluación de alter- nativas}

En la Figura 19 se presentan las alternativas que se han identificado como posibles para el control hidrogeológico durante las operaciones de la Fase 1B. Esas alternativas son las que habitualmente se contemplan en la práctica hidrogeológica aplicada a la geotecnia y la minería (Arroyo Álvarez de Toledo and Gens Solé, 2008; Carrera and Vázquez-Suñé, 2008; Niñerola Pla, 2008).

Las alternativas posibles y sus características fundamentales son las siguientes:

- Achique del agua desde el fondo de la excavación.
- Extracción de los residuos bajo lámina de agua.

- Rebaje de los niveles piezométricos mediante pozos o zanjas.

- Construcción de una barrera perimetral estanca.

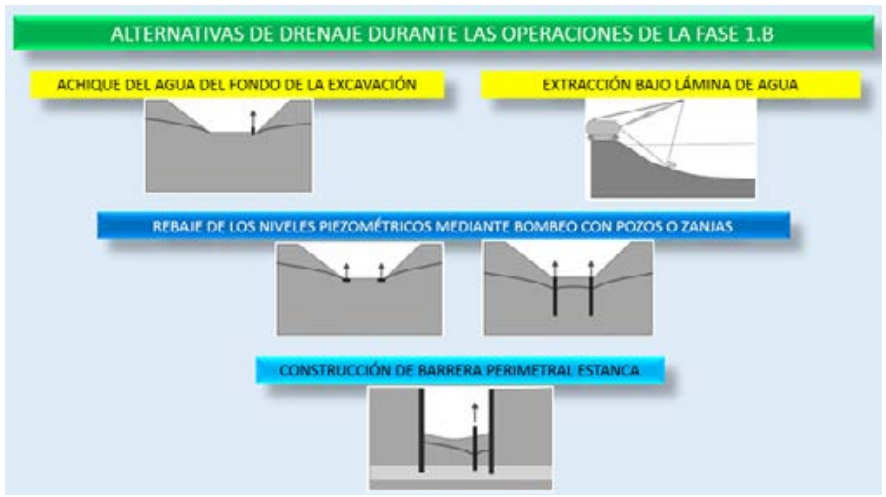

Figura 19. Alternativas de control hidrogeológico durante las operaciones de drenaje de la Fase 1.B.

Figure 19. Hydrogeological control options during Phase 1.B drainage operations.

\section{Achique del agua desde el fondo de la excavación}

Consiste en el bombeo al exterior de la excavación del agua subterránea que vaya fluyendo hacia la balsa durante las operaciones de extracción de los residuos. El agua bombeada deberá ser tratada previamente a su vertido en cauce público, lo que requerirá la autorización por la CHTajo que exigirá los correspondientes estándares de calidad. 


\section{Extracción de los residuos bajo lámina de agua}

No se precisa la utilización de bombas para el rebaje de la lámina de agua porque utilizando maquinaria pesada y potente se va extrayendo todo el material del interior de la balsa. No hay un control de las operaciones de excavación debido a que el cazo empleado se sumerge sistemáticamente bajo la lámina de agua y por lo tanto el material de excavación que se obtenga debe ser convenientemente separado, produciéndose un empeoramiento progresivo de la calidad del agua que deberá ser corregido posteriormente con el tratamiento adecuado.

Rebaje de los niveles piezométricos mediante pozos o zanjas

Aplicando el rebaje de los niveles piezométricos la operación puede realizarse teóricamente en seco, si bien los caudales de agua subterránea que se extraigan deben ser tratados para su retorno a cauce público en las condiciones que requiera la $\mathrm{CHTajo,} \mathrm{según}$ la autorización que sea otorgada por ese organismo.

\section{Construcción de una barrera perimetral estanca}

El diseño de la barrera perimetral de paredes estancas tiene como objeto aislar la zona de extracción de los residuos del resto del acuífero. La oportunidad de esta alternativa viene determinada por las características del subsuelo investigadas en la fase de anteproyecto y que pusieron de manifiesto la presencia de una capa arcillosa de $2 \mathrm{~m}$ de espesor (TRAGSA and EMGRISA, 2013). Para ser efectiva el muro impermeable de la barrera perimetral debe ser empotrado en esta capa arcillosa infrayacente.

En los dos próximos apartados se describen los resultados obtenidos de las alternativas y la selección de la alternativa óptima.

\section{Resultados: Caracterización de alternativas}

\section{Achique del agua desde el fondo de la excavación}

En esencia este sistema consiste en la ubicación en el fondo de la excavación de una o varias bombas de achique que permitan mantener en seco la zona de trabajo.

En la Figura 20 se representa las condiciones de flujo subterráneo para poner de manifiesto tanto el gradiente piezométrico como el caudal de surgencia a través de los limos infrayacentes a los residuos depositados en el fondo de la balsa.

En caso de achique directo con bomba, el gradiente hidráulico podría alcanzar un valor muy elevado de hasta 7 (3.5 metros de diferencia piezométrica en 0.5 $\mathrm{m}$ de espesor de limos). Además, la carga hidráulica considerada de $4 \mathrm{~m}$ corresponde al momento más favorable del periodo de estiaje.

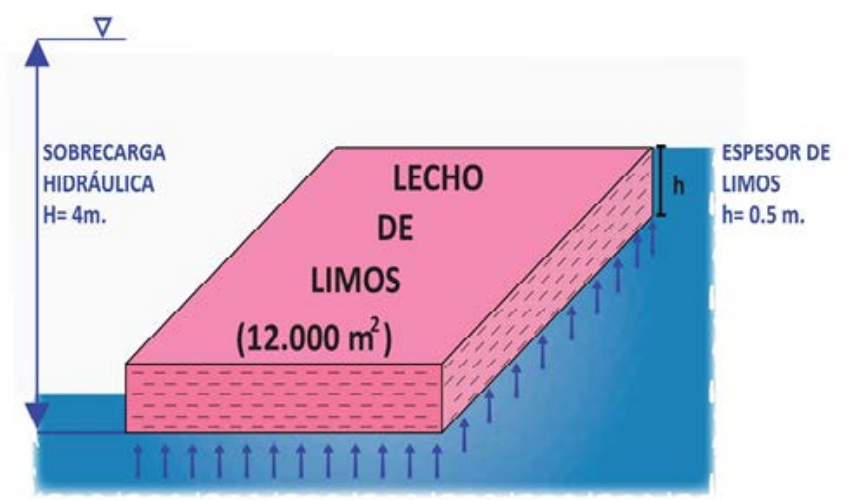

Figura 20. Sobrecarga hidráulica en caso de realizar achique directo con bomba durante la extracción por medios mecánicos del residuo.

Figure 20. Hydraulic overload in case of direct bilge with pump during mechanical residue extraction.

Para evaluar los caudales de surgencia se aplica la Ley de Darcy, según la cual, considerando un gradiente de 7 , sobre una superficie de $12000 \mathrm{~m}^{2}$ y una permeabilidad de limos de $2 \times 10^{-7} \mathrm{~m} / \mathrm{s}$, resulta un caudal en continuo de $16.79 \mathrm{l} / \mathrm{s}$.

El sistema de desagüe directo con bombas se desaconseja totalmente en aquellas excavaciones donde en su fondo se puedan producir elevados gradientes piezométricos, que puedan superar el gradiente crítico provocando efectos de sifonamiento con arrastres, roturas y cambios en la capacidad portante del terreno. Puesto que el gradiente hidráulico crítico tiene el valor de 1 , teniendo en cuenta el valor de 7 que pudieran generarse, las operaciones de achique directo de la balsa no son aconsejables en estas condiciones.

\section{Extracción de los residuos bajo lámina de agua}

Este sistema es habitualmente empleado en las graveras que explotan áridos y no se produce un drenaje como tal de la lámina de agua. Una ventaja importante de este método es que no se requiere el empleo de sistemas de bombeo. Otra ventaja es que las operaciones de extracción se realizan de forma sistemática a un ritmo alto, pero su empleo, debido a la falta de control directo de las operaciones extractivas, genera muchas incógnitas, algunas de difícil previsión y cuantificación.

Entre las incógnitas que se presentan está la afección que pueda producirse sobre el acuífero por la falta de control sobre la acción de los limos situados bajo los residuos, la posibilidad de mezcla de los residuos con el acuífero infrayacente, la necesidad de separación posterior de terreno y residuos, así como el secado de los mismos. Además, otro efecto a considerar es la necesidad de uso de maquinaria pesada que debería transitar sobre unos terrenos bastante inestables, debido al porcentaje de residuos y rellenos poco compactados. 


\section{Rebaje de los niveles piezométricos mediante pozos o zanjas}

En este apartado se presentan los resultados obtenidos sobre los volúmenes y caudales de extracción de aguas subterráneas en pozos o zanjas a partir de cuatro disposiciones:

- Drenaje lineal mediante batería de 12 pozos.

- Drenaje lineal mediante zanja de 120 metros.

- Sistema de drenaje perimetral con anillo de 43 pozos.

- Sistema de drenaje perimetral con zanja.

a. Drenaje lineal mediante batería de 12 pozos o zanja de $120 \mathrm{~m}$

Se ha simulado una disposición lineal de drenaje (pozos o zanjas) que teóricamente permitiría la extracción de los residuos de forma progresiva alternando la extracción parcial, el relleno simultáneo y el avance hacia el interior de la laguna según las operaciones que se recogen en la Figura 21. Las alternativas de la operación serían:

- Hacer bermas escalonadas a partir del perfil del talud de la balsa, retirando la costra contaminante, impermeabilizando con arcilla y relle-
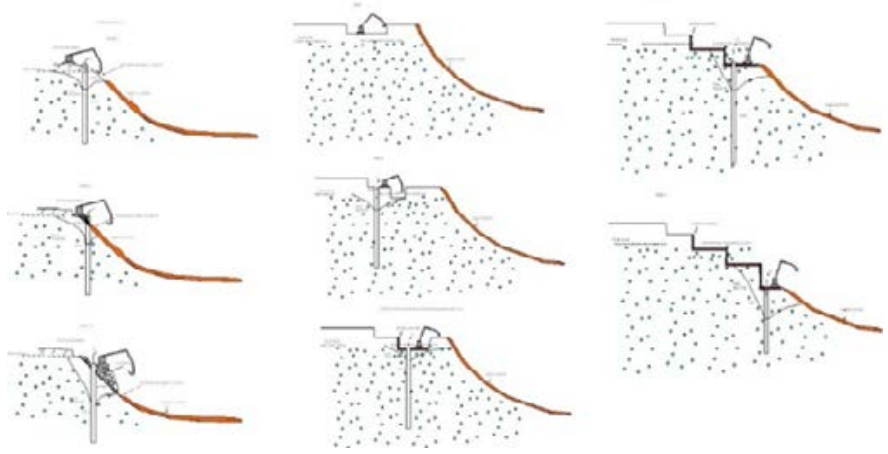

Figura 21. Alternativas de excavación y rebaje de taludes con un sistema lineal de drenaje con bombeo en pozos.

Figure 21. Options for excavation and slope reduction with a linear drainage system with well pumping.
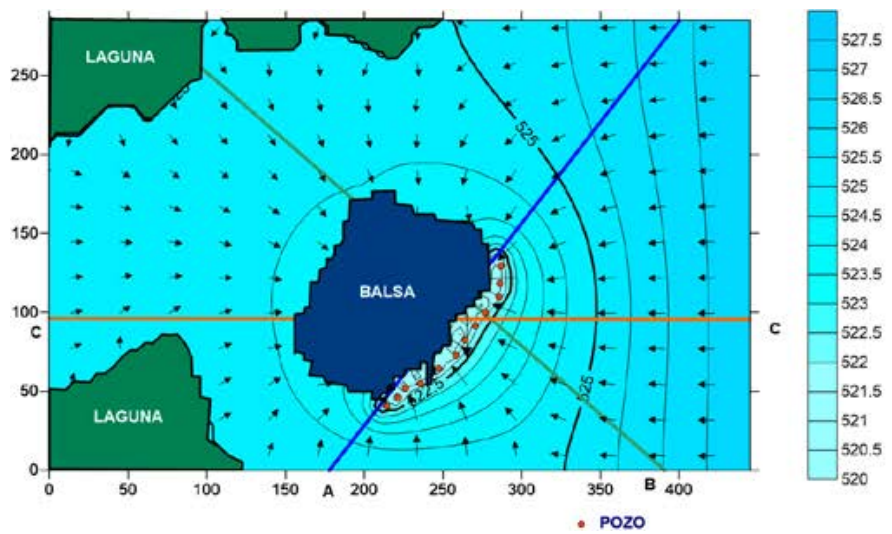

nando con bloques de escollera y arena, para después hacer nuevos pozos en las bermas construidas, ya que los radios de influencia efectivos son pequeños.

- Rebajar la pendiente del talud haciendo escalones desde los cuales se retire la costra contaminante y se impermeabilice con arcillas y pantallas.

En la Figura 22 se presenta la disposición de la batería de 12 pozos que constituyen el drenaje lineal con bombeo.

En la Figura 23 se recogen las superficies piezométricas obtenidas, de acuerdo con la modelización realizada con el código MODFLOW, tanto para la batería de pozos como para la zanja de $120 \mathrm{~m}$.

b. Sistema de drenaje perimetral con anillo de 43 pozos o zanja

El sistema de drenaje perimetral es el método que habitualmente se utiliza para trabajar en seco en una explotación minera a cielo abierto, bien sea mediante bombeo con pozos (explotaciones profundas) o zanjas de drenaje (explotaciones de minería superficiales, como las graveras).

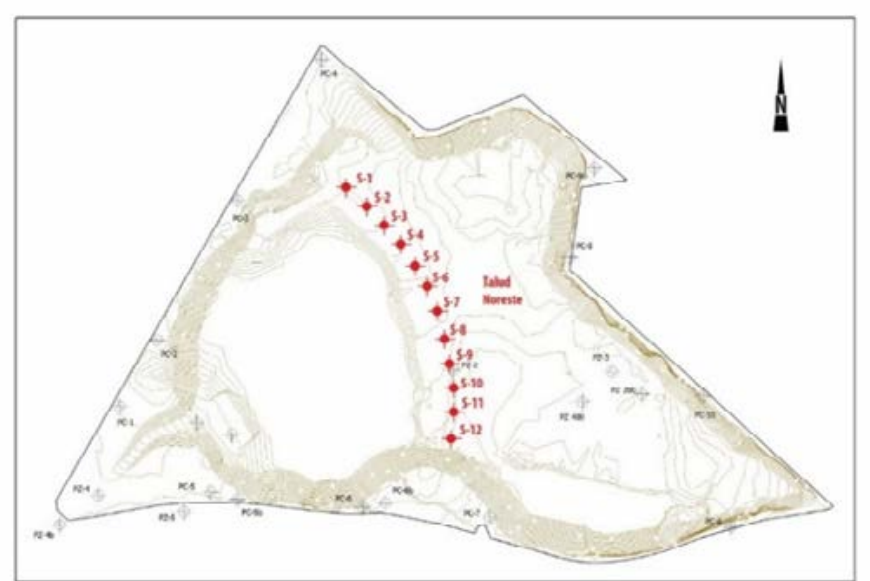

Figura 22. Disposición del drenaje lineal mediante una batería de 12 pozos.

Figure 22. 12-well battery linear drainage.

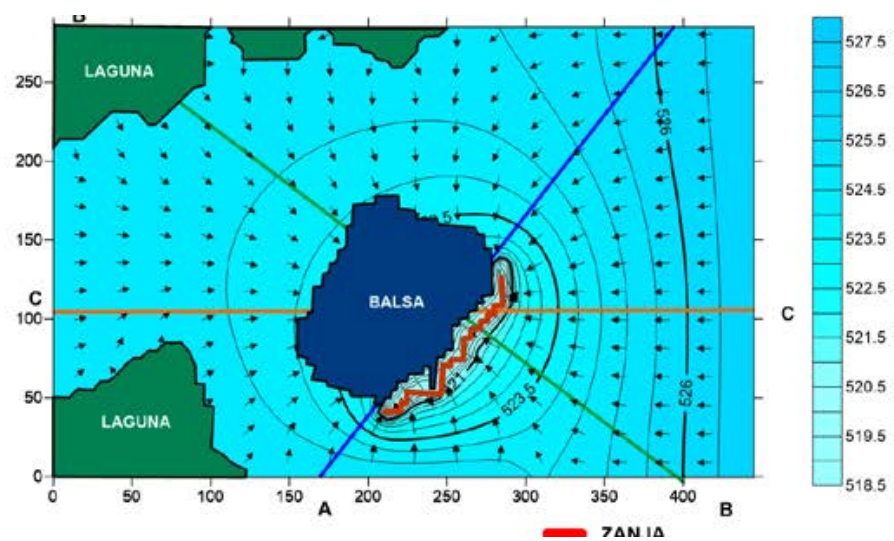

Figura 23. Superficies piezométricas en la simulación de drenaje lineal con batería de pozos y zanja, respectivamente. Figure 23. Piezometric surfaces in the simulation of linear drainage with battery wells and ditch, respectively. 
En la Figura 24 se presenta las superficies piezométricas obtenidas con los sistemas de drenaje perimetral, resultado de deprimir los niveles a la cota $522 \mathrm{~m}$ s.n.m.

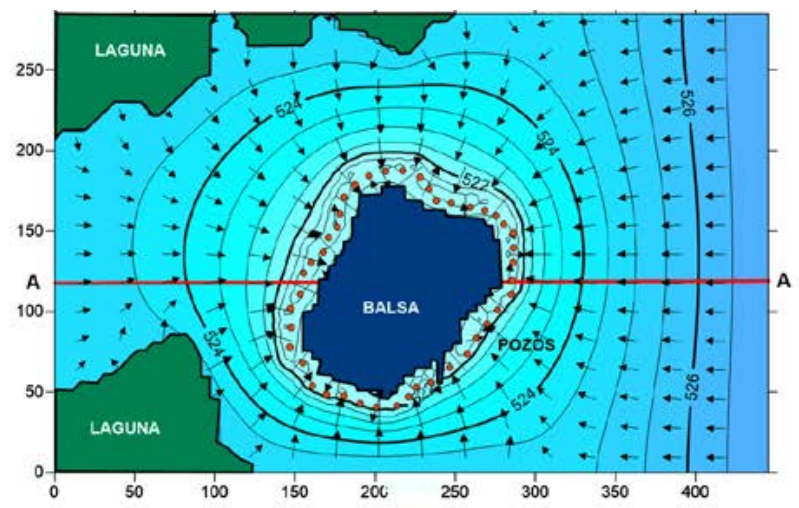

c. Cuantificación de los caudales y volúmenes de drenaje

En la Tabla 6 se recogen de forma resumida los va-

Figura 24. Superficies piezométricas en la simulación de drenaje perimetral con anillo de pozos y zanja, respectivamente.

Figure 24. Piezometric surfaces in the simulation of perimeter drainage with ring of wells and ditch, respectively.

\begin{tabular}{|c|c|c|c|c|}
\hline $\begin{array}{c}\text { Caudal por pozo } \\
(1 / \mathbf{s}) \\
\end{array}$ & $\begin{array}{c}\text { Caudal Total } \\
(\mathrm{l} / \mathrm{s}) \\
\end{array}$ & $\begin{array}{c}\text { Volumen diario } \\
\left(\mathrm{m}^{3}\right) \\
\end{array}$ & $\begin{array}{c}\text { Volumen } \\
\text { trimestral }\left(\mathrm{m}^{3}\right)\end{array}$ & $\begin{array}{c}\text { Volumen anual } \\
\left(\mathrm{m}^{3}\right) \\
\end{array}$ \\
\hline \multicolumn{5}{|c|}{ Dre naje lineal mediante batería de 12 pozos } \\
\hline \multicolumn{5}{|c|}{ Acuífero único interconectado } \\
\hline 15 & 180 & 15552 & 1399680 & 5598720 \\
\hline \multicolumn{5}{|c|}{ Acuífero superficial desconectado en las arcillas } \\
\hline 2.5 & 30 & 2592 & 233280 & 933120 \\
\hline \multicolumn{5}{|c|}{ Dre naje lineal mediante zanja de $120 \mathrm{~m}$ de longitud } \\
\hline \multicolumn{5}{|c|}{ Acuífero único interconectado } \\
\hline- & 238 & 20563 & 1850688 & 7402752 \\
\hline \multicolumn{5}{|c|}{ Acuifero superficial desconectado en las arcillas } \\
\hline- & 32 & 2765 & 248832 & 995328 \\
\hline \multicolumn{5}{|c|}{ Dre naje perime tral con anillo de 43 pozos } \\
\hline \multicolumn{5}{|c|}{ Acuífero único interconectado } \\
\hline Entre 7 y 9 & 376 & 32486 & 2923775 & 11695104 \\
\hline \multicolumn{5}{|c|}{ Acuífero superficial desconectado en las arcillas } \\
\hline Entre 1.3 y 1.7 & 71.9 & 6212 & 559004 & 2236378 \\
\hline \multicolumn{5}{|c|}{ Acuífero somero desconectado en los limos } \\
\hline Entre 1 y 1.5 & 57 & 4925 & 443232 & 1772928 \\
\hline \multicolumn{5}{|c|}{ Drenaje perimetral con zanja } \\
\hline \multicolumn{5}{|c|}{ Acuifero único interconectado } \\
\hline- & 455 & 39312 & 3538080 & 14152320 \\
\hline \multicolumn{5}{|c|}{ Acuifero superficial desconectado en las arcillas } \\
\hline- & 72.8 & 6290 & 566093 & 2264371 \\
\hline \multicolumn{5}{|c|}{ Acuífero somero desconectado en los limos } \\
\hline- & 63,33 & 5472 & 492454 & 1969816 \\
\hline
\end{tabular}

Tabla 6. Valores obtenidos con MODFLOW para distintas hipótesis de drenaje perimetral y descenso piezométrico a la cota $522 \mathrm{~m}$ s.n.m. Table 6. Values obtained with MODFLOW for different hypotheses of perimeter drainage and piezometric descent at the $522 \mathrm{~m}$ a.s.l. 
lores de caudales y volúmenes que se han obtenido en la simulación con MODFLOW y que es necesario drenar para conseguir un descenso piezométrico a la cota de 522 m s.n.m. en el interior de la balsa de residuos.

A la vista de laTabla 6 se pone de manifiesto la elevada magnitud de los caudales y volúmenes que requiere el drenaje con bombeo en pozos o con zanjas, independientemente del sistema de drenaje que se utilice. Esta circunstancia hace muy problemático el empleo de esta alternativa para poder trabajar en seco, dado el volumen de agua subterránea a extraer, el efecto causado por de arrastre de sustancias no deseables durante el drenaje y los estándares de calidad que exige la legislación vigente para la reincorporación al medio hídrico del agua subterránea extraída. Las instalaciones de tratamiento necesarias, con volúmenes muy elevados y gran variedad de sustancias a tratar tendrían un coste desorbitado, al que habría que añadir el de la construcción de los sistemas de bombeo e impulsión. Además de los condicionantes económicos, las características geotécnicas del subsuelo, con poca estabilidad debida a la gran cantidad de rellenos y residuos, son muy adversas para hacer fluir grandes caudales de agua subterránea a su través.

\section{Construcción de una barrera perimetral estanca}

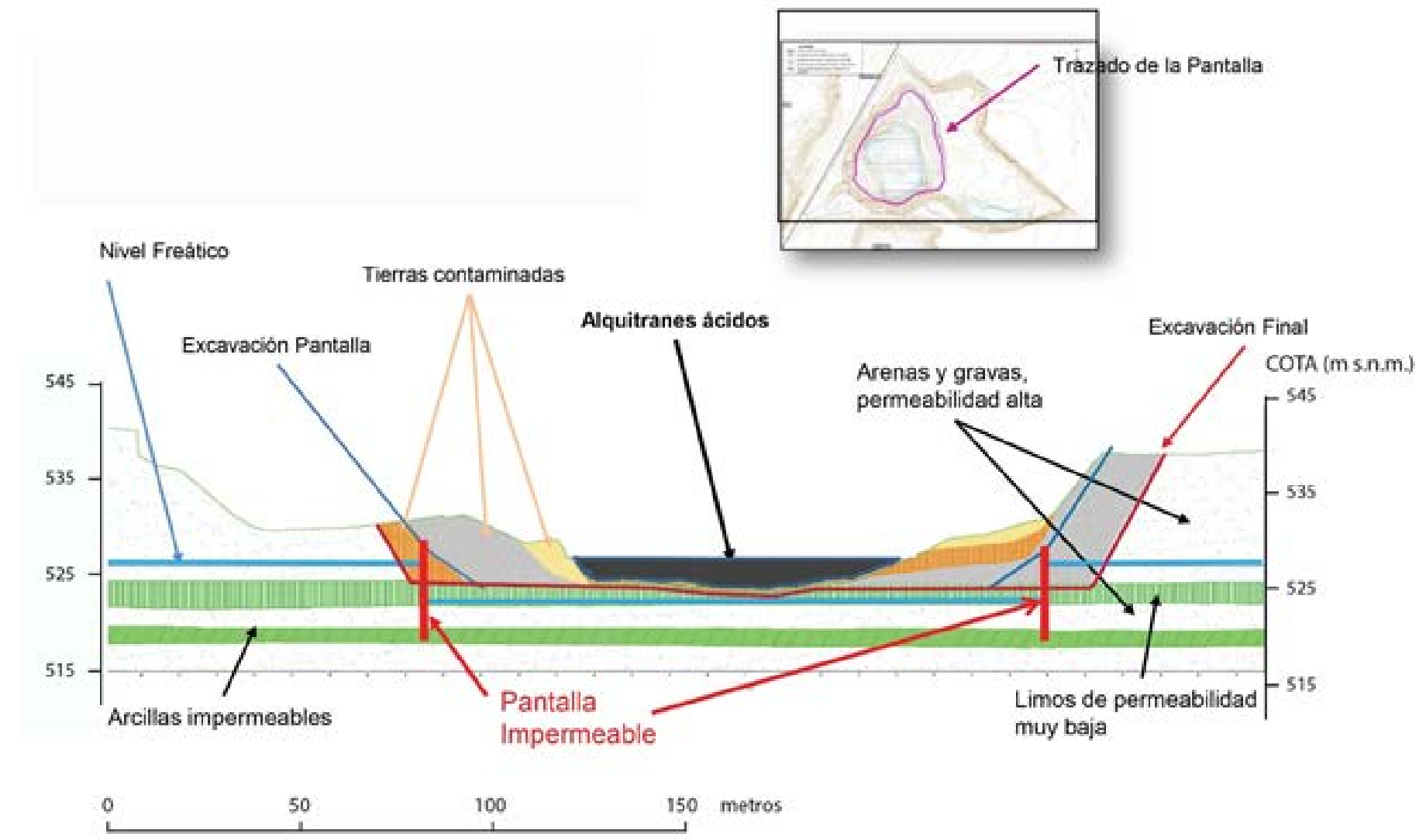

Figura 25. Perfil de la pantalla sellante proyectada, anclada en las arcillas infrayacentes y rebajamiento del nivel freático. Figure 25. Profile of the projected sealed screen, anchored in the underlying clay and the lowering of the water table.
El sistema de impermeabilización mediante la construcción de una barrea perimetral estanca consiste en la construcción de una pantalla perimetral sellante, desde superficie y hasta una profundidad tal que se empotre en niveles litológicos existentes en el subsuelo del terreno que sean impermeables como son las arcillas detectadas en el perfil. Estas pantallas impermeables para controlar el agua bombeada se utilizan habitualmente en edificación cuando es preciso efectuar excavaciones de cierta profundidad bajo el nivel freático en materiales geotécnicamente deficientes. Los materiales que más habitualmente se utilizan para la impermeabilización son los muros pantalla de hormigón, los tablestacados y las pantallas de lechada de bentonita, en función de las características de resistencia de cimentación y de contención que son requeridas.

En principio este es un sistema muy interesante y adecuado para utilizar durante la extracción del residuo de la balsa, pues, acorde con los datos considerados en el anteproyecto (TRAGSA and Emgrisa, 2013), en el subsuelo de la parcela existe un nivel arcilloso de $2 \mathrm{~m}$ de espesor comprendido entre 519 y $517 \mathrm{~m}$ s.n.m. que presenta una permeabilidad muy baja $(2,5$ $\times 10^{-9} \mathrm{~m} / \mathrm{s}$ ), que es propia de un material sellante, adecuado poder empotrar la barrera impermeable. En la Figura 25 se muestra la disposición y desarrollo de la barrera perimetral impermeable. 
Una vez construida la barrera estanca se procederá a la extracción del agua subterránea presente en el espacio confinado, rebajando el nivel piezométrico hasta una cota de seguridad de $521 \mathrm{~m}$ s.n.m. Alcanzada esta cota podrá comenzarse la Fase 1B para la extracción de los residuos por medios mecánicos, pues la excavación interior a la barrera se efectuará en condiciones teóricas de estanqueidad y en seco.

La cuantificación de los volúmenes a extraer se ha calculado directamente mediante formulación analítica. En primer lugar, se ha calculado el volumen teórico que sería necesario extraer, bajo supuesto de impermeabilización total de las paredes de la pantalla, para rebajar el nivel a la cota piezométrica por debajo de la cota de muro de la capa de limos infrayacentes a la balsa (521 m s.n.m.). Posteriormente se ha aplicado la Ley de Darcy para ver el caudal que sería necesario bombear desde el interior del recinto y extraer el caudal de infiltración que pudiera circular a través de las arcillas. También se han calculado los volúmenes de agua que podrían eliminarse por evaporación directa en la lámina libre de una balsa de evaporación de $2000 \mathrm{~m}^{2}$ de superficie que se construyera a los efectos en la parcela.

Asumiendo la hipótesis de estanqueidad total, el volumen liberado al deprimir en un recinto estanco un nivel piezométrico es el producto del área, el coeficiente de almacenamiento y la depresión del nivel piezométrico. Para deprimir el nivel piezométrico de la balsa es preciso rebajar el nivel piezométrico en dos fases, una primera entre las cotas $526 \mathrm{~m}$ s.n.m. y la 521,5 m s.n.m. en las que el acuífero se comporta

\begin{tabular}{|c|c|c|c|}
\hline $\begin{array}{c}\text { Volumen de } \\
\text { evacuación }\left(\mathrm{m}^{3}\right)\end{array}$ & $\begin{array}{c}\text { Volumen diario de } \\
\text { extracción }\left(\mathrm{m}^{3}\right)\end{array}$ & $\begin{array}{l}\text { Volumen trimestral } \\
\text { de extracción }\left(\mathrm{m}^{3}\right)\end{array}$ & $\begin{array}{c}\text { Volumen anual de } \\
\text { extracción }\left(\mathrm{m}^{3}\right)\end{array}$ \\
\hline 1827 & 5 & 450 & 1825 \\
\hline
\end{tabular}

Tabla 7. Valores calculados sobre los volúmenes de evacuación y de extracción bajo hipótesis de pantalla empotrada en la capa de arcilla infrayacente al subsuelo de la balsa.

Table 7. Calculated volumes of evacuation and extraction under the hypothesis of a waterproof barrier in the clay layer below the base of the raft.

\begin{tabular}{|c|c|c|c|}
\hline Acción & Ventajas & Inconvenientes & Decisión \\
\hline $\begin{array}{l}\text { Achique del agua desde el } \\
\text { fondo de la excavación }\end{array}$ & Sistema simple de implementar & $\begin{array}{l}\text { Problemas graves de sifonamiento } \\
\text { Caudales importantes a depurar } \\
\text { Movilización de sustancias } \\
\text { presentes en el subsuelo }\end{array}$ & Descartada \\
\hline $\begin{array}{l}\text { Extracción de los residuos } \\
\text { bajo lámina de agua }\end{array}$ & $\begin{array}{l}\text { No requiere sistemas de bombeo } \\
\text { Extracción sistemática con buen ritmo }\end{array}$ & $\begin{array}{l}\text { Falta de control sobre la posible } \\
\text { afección al acuifero } \\
\text { Riesgo de mezcla de residuos } \\
\text { con el terreno acuífero } \\
\text { Utilización de maquinaria muy } \\
\text { pesada en terrenos poco estables }\end{array}$ & Descartada \\
\hline $\begin{array}{l}\text { Rebaje de los niveles } \\
\text { piezométricos mediante } \\
\text { pozos o zanjas }\end{array}$ & $\begin{array}{l}\text { Eficiencia, flexibilidad } \\
\text { y control del sistema }\end{array}$ & $\begin{array}{l}\text { Caudales muy elevados a bombear } \\
\text { Movilización muy alta de sustancias } \\
\text { presentes en el subsuelo } \\
\text { Necesidad de elevadas inversiones } \\
\text { en planta depuradora }\end{array}$ & Descartada \\
\hline & & $\begin{array}{l}\text { Inversión importante para } \\
\text { implementar los sistemas de drenaje } \\
\text { Gestión compleja de autorizaciones } \\
\text { administrativas }\end{array}$ & \\
\hline $\begin{array}{l}\text { Construcción de una } \\
\text { barrera perimetral estanca }\end{array}$ & $\begin{array}{l}\text { Evita bombeo de volúmenes } \\
\text { importantes de agua } \\
\text { No produce grandes movilizaciones } \\
\text { de las sustancias } \\
\text { Gestión simple de autorizaciones } \\
\text { administrativas } \\
\text { En teoria no se requiere inversión en } \\
\text { sistemas de depuración } \\
\text { Sistemas de bombeo muy sencillos }\end{array}$ & $\begin{array}{l}\text { Coste de construcción de la barrera } \\
\text { Necesidad de obtener estanqueidad } \\
\text { en arcillas y barrera }\end{array}$ & Seleccionada \\
\hline
\end{tabular}

Tabla 8. Matriz de decisión para seleccionar la alternativa más ventajosa.

Table 8. Decision matrix to select the most advantageous option. 
como confinado, por el nivel sellante de los limos, y el coeficiente de almacenamiento sería del orden de $5 \times 10^{-4}$, siendo el volumen resultante a extraer de 27 $\mathrm{m}^{3}$. En la segunda fase entre las cotas $521,5 \mathrm{~m}$ s.n.m. (muro del acuífero), y 521 m s.n.m. el acuífero se comporta como libre, bajo el nivel sellante de los limos, y el coeficiente de almacenamiento sería del orden de unos $30 \times 10^{-2}$, correspondiendo un volumen a extraer de $1800 \mathrm{~m}^{3}$. Para calcular el caudal de circulación a través de las arcillas, una vez se hubiera impermeabilizado con una pantalla perimetral sellante extendida hasta dicha capa de arcillas, se puede aplicar directamente la ley de Darcy para un área de paso de $12000 \mathrm{~m}^{2}$ con una permeabilidad de $2.5 \times 10^{-9} \mathrm{~m} / \mathrm{s}$ y un gradiente piezométrico de 2, resulta un volumen de 5 $\mathrm{m}^{3} /$ día. En la tabla 7 se recogen los resultados calculados, bajo la hipótesis de estanqueidad del sistema formado por la pantalla impermeable empotrada en la capa de arcilla infrayacente.

A partir de los valores medios de evaporación en embalses de la Cuenca del Tajo y el incremento con respecto a la media durante los meses más secos (Témez Peláez, 2007) se considera que la evaporación diaria en la zona de la laguna de Arganda puede ser del orden de $5 \mathrm{~mm} /$ día. Por lo tanto, durante los tres meses de estiaje en los que se llevarían a cabo las operaciones de extracción de los residuos el volumen teórico que podría eliminarse por evaporación directa sobre lámina libre para una superficie de $2000 \mathrm{~m}^{2}$ sería de $10 \mathrm{~m}^{3}$ al día.

Puede concluirse que una balsa excavada e impermeabilizada con una superficie de $2000 \mathrm{~m}^{2}$ y una profundidad de $1 \mathrm{~m}$ en teoría podría permitir gestionar la extracción del agua subterránea bombeada para realizar las operaciones de extracción de residuos en seco, evitando la necesidad de un bombeo de gran caudal de aguas subterráneas y la posterior depuración y vertido al exterior. Por lo tanto, de acuerdo con la legislación de aguas, no sería necesaria la tramitación y obtención de permisos ante la CHTajo, a excepción del correspondiente al escaso volumen bombeado, para lo que, en rigor, sería suficiente, teóricamente, con una mera autorización de menos de $7000 \mathrm{~m}^{3}$ al año, evitando así la tramitación mucho más compleja de una concesión que la normativa requiere para volúmenes anuales de extracción superiores al indicado.

\section{Discusión y selección de la alternativa óptima}

Una vez realizada la identificación y caracterización de alternativas se ha procedido a la selección de la que se considera óptima. Para ello se ha elaborado la matriz de decisión (Tabla 8), en la que se recogen las ventajas e inconvenientes de cada una de ellas de forma que se pueda adoptar una decisión razonada basada en criterios lógicos.

A la vista del análisis realizado en dicha matriz de decisión, se considera que la opción más ventajosa es la construcción de una barrera perimetral estanca.

\section{Conclusiones}

El sistema de impermeabilización mediante pantalla sellante, anclada en la capa de arcillas infrayacente, se presenta como el sistema más adecuado para el control de las aguas subterráneas durante las próximas operaciones de extracción por medios mecánicos de los residuos del fondo de la balsa que no son susceptibles de bombeo, lo que constituye la Fase 1B de acuerdo, con la planificación de las labores de restauración de la Laguna de Arganda del Rey (Madrid).

Esta alternativa, aunque supone el costo de construcción de la pantalla, presenta un ahorro muy importante en los conceptos de bombeo, tratamiento de depuración y vertido corregido a cauce público de elevadísimos volúmenes de agua y también evita una compleja y laboriosa tramitación ante la Confederación Hidrográfica del Tajo. De acuerdo con los cálculos realizados, en las condiciones de estanqueidad deducidas de la caracterización del subsuelo de los estudios previos realizados, los volúmenes de agua a extraer son muy bajos y es técnicamente posible la eliminación de los mismos mediante evaporación en una balsa exterior del agua bombeada.

Una vez finalizada la barrera impermeable anclada en las arcillas será preciso construir en su interior los pozos de evacuación y drenaje y los correspondientes piezómetros para verificar la estanqueidad del sistema. Durante las operaciones de extracción de la Fase 1B. es necesario el control piezométrico y de calidad en la red perimetral y las lagunas adyacentes, así como de los pozos y piezómetros de control de la barrera.

\section{Agradecimientos}

Los autores quieren mostrar su agradecimiento a la Consejería de Medio Ambiente, Ordenación del Territorio y Sostenibilidad de la Comunidad de Madrid, a la Universidad Politécnica de Madrid, a las empresas TRAGSA y CGSi por la información y las facilidades dadas para la preparación de este trabajo.

\section{Referencias}

Arroyo, M. and Gens, A. 2008. Acoplamiento hidromecánico en excavaciones. Jornadas Técnicas El Agua y las infraestructuras en el medio subterráneo. Barcelona, 1-20.

Carrera, J. and Vázquez-Suñé, E. 2008. Sobre la interacción entre acuíferos y obras subterráneas. Jornadas Técnicas El Agua y las infraestructuras en el medio subterráneo. Barcelona, 21-38.

Centro de Descargas de Infraestructura de Datos Espaciales (IDE). Ministerio para laTransición Ecológica, 04/05/17, https:/www.miteco.gob.es/es/cartografia-y-sig/ $\mathrm{ide/descargas/agua/masas-de-agua-phc-2015-2021.aspx.}$

Ecología acuática. Consejo Superior de Investigaciones Científicas (CSIC), 31/05/2017, http://www.hu- 
medalesibericos.com

Mora, P., Castilla, J., Herrera, J., Barettino, D. 2017. Ecological restoration of a former gravel pit contaminated by a massive petroleum sulfonate spill. A case study: Arganda del Rey. Madrid (Spain). Ecological engineering, 100, 73-88.

Niñerola, J.M. 2008. El control hidrogeológico para la evaluación de los impactos de los proyectos y actividades en los acuíferos. Caso del Llobregat Jornadas Técnicas El Agua y las infraestructuras en el medio subterráneo. Barcelona, 355-364.

Parque Regional del Sureste. Consejería de Medio Ambiente y Ordenación del Territorio de la Comunidad de Madrid, 31/05/17, http://www.parqueregionalsureste.org.

Témez, J.R. 2007. Consideraciones prácticas sobre la evaporación en los embalses de la España peninsular. Revista de Obras Públicas 3476, 15-22.

TRAGSA and CGSi 2017a. Red de circulación de las aguas subterráneas en el subsuelo de la parcela de estudio. Informe Inédito. Dirección General de
Economía Circular de la Comunidad de Madrid.

TRAGSA and CGSi 2017b. Cálculo de los caudales de drenaje con MODFLOW. Informe Inédito. Dirección General de Economía Circular de la Comunidad de Madrid.

TRAGSA and CGSi 2017c. Evaluación de las alternativas de control de las aguas subterráneas. Informe Inédito. Dirección General de Economía Circular de la Comunidad de Madrid.

TRAGSA and EMGRISA 2013. Anteproyecto de Recuperación Ambiental de las parcelas $146 \mathrm{C}$ y D del polígono 47 de Arganda del Rey. Informe Inédito. Dirección General de Economía Circular de la Comunidad de Madrid.

US Geological Survey 2016. MODFLOW and related programs. https://www.usgs.gov/mission-areas/ water-resources/science/modflow-and-related-programs.

Visor CHTajo. Confederación Hidrográfica del Tajo (CHTajo), 31/05/17, http://www.chtajo.es/LaCuenca/ Paginas/visor.aspx.

Recibido: julio 2020

Revisado: diciembre 2020

Aceptado: enero 2021

Publicado: diciembre 2021 
